\title{
Impact of tumor-infiltrating LAMP-3 dendritic cells on the prognosis of esophageal squamous cell carcinoma
}

Junya Nishimura, Hiroaki Tanaka, Yoshihito Yamakoshi, Soichiro Hiramatsu, Tatsuro Tamura, Takahiro Toyokawa, Kazuya Muguruma, Kiyoshi Maeda, Kosei Hirakawa \& Masaichi Ohira

\begin{tabular}{|c|l|}
\hline Citation & Esophagus. $16 ; 333-344$ \\
\hline Issue Date & $2019-04-09$ \\
\hline Type & Journal Article \\
\hline Textversion & author \\
\hline Relation & $\begin{array}{l}\text { This is a post-peer-review, pre-copyedit version of an article published in Esophagus. } \\
\text { The final authenticated version is available online at: } \\
\text { https://doi.org/10.1007/s10388-019-00669-w. }\end{array}$ \\
\hline DOI & $10.1007 / \mathrm{s} 10388-019-00669-\mathrm{w}$ \\
\hline
\end{tabular}

Self-Archiving by Author(s) Placed on: Osaka City University

Nishimura, J., Tanaka, H., Yamakoshi, Y. et al. Impact of tumor-infiltrating LAMP-3 dendritic cells on the prognosis of esophageal squamous cell carcinoma. Esophagus 16, 333-344 (2019). doi: 10.1007/s10388-019-00669-w 
Impact of tumor-infiltrating LAMP-3 dendritic cells on the prognosis of

esophageal squamous cell carcinoma

Junya Nishimura, Hiroaki Tanaka, Yoshihito Yamakoshi, Soichiro Hiramatsu, Tatsuro

Tamura, Takahiro Toyokawa, Kazuya Muguruma, Kiyoshi Maeda, Kosei Hirakawa,

Masaichi Ohira

Department of Surgical Oncology, Osaka City University Graduate School of Medicine,

Osaka, Japan

Corresponding author: Hiroaki Tanaka; Asahimachi 1-4-3 Abenoku Osaka, Japan;

m1351176@med.osaka-cu.ac.jp 


\section{Abstract.}

Background: Dendritic cells (DCs) are the most potent antigen-presenting cells to induce cytotoxic $\mathrm{T}$ lymphocytes in the tumor environment. After acquiring antigens, DCs undergo maturation and their expression of $\mathrm{MHC}$ and co-stimulation molecules are enhanced, along with lysosome-associated membrane glycoprotein 3 (LAMP-3), which is a specific marker of mature DCs. In general, mature DCs are usually considered to be immunostimulatory in the cancer microenvironment. In addition, it is known that tumorinfiltrating lymphocytes (TILs) are associated with a good prognosis in esophageal squamous cell carcinoma (ESCC). However, few studies have targeted the interaction between DCs and TILs in the local immunity of ESCC. We investigated the localization of mature DCs within ESCC tissue and their relationship to TILs as well as the clinical outcome.

Methods: We evaluated 80 ESCC patients who underwent surgical treatment without preoperative treatment, using immunohistochemistry with LAMP-3 and CD8.

Results: The results showed that LAMP-3 DCs were predominantly observed in the peritumoral area. Intratumoral CD8 T cells were found to be associated with a favorable prognosis, and the number of infiltrating LAMP-3 DCs was correlated with the number of intratumoral CD8 T cells. 
Conclusion: At the local tumor site, mature LAMP-3 DCs might be associates with increasing tumor infiltrating CD8 T cells.

Key words: esophageal, dendritic cell, LAMP-3 


\section{Introduction}

Esophageal squamous cell carcinoma (ESCC) is one of the most aggressive cancers in Asia [1]. As some investigators have demonstrated the effectiveness of immunotherapy for squamous cell carcinoma, the importance of the immune response at the local site has come into focus [2]. Tumor-infiltrating immune cells are important components of the tumor microenvironment and are associated with the prognosis of cancer patients [3]. Among these, tumor-infiltrating lymphocytes (TILs), including CD4 and CD8 T cells, are associated with a good clinical outcome in many different types of tumor, including ESCC [4] [5] [6]. It is suggested that infiltrating CD4 T cells help prime CD8 T cells, which have anti-tumor cytotoxic capacity and can persist in vivo over several years as memory $\mathrm{T}$ cells [7]. In the context of tumor immunity, the interaction of $\mathrm{T}$ cells with mature dendritic cells (DCs) generates central memory and effector T cells [8].

Mature DCs present exogenous and endogenous antigens on major histocompatibility class molecules(MHCs) to bind to T cell receptors [9]. DC maturation is represented by the enhanced expression of MHCs, co-stimulatory molecules and lysosome-associated membrane glycoprotein 3 (LAMP-3) [10]. LAMP-3 is a member of the lysosomeassociated membrane glycoprotein family and was found in the MHC class II compartment immediately before the translocation of MHC class II molecules to the cell surface [11]. LAMP-3 appears transiently on DC activation at the limiting membrane of 
the MHC class II-containing intracellular compartments (MIICs) involved in MHC class II peptide loading and transport to the cell surface [11] [12]. Therefore, LAMP-3 is regarded as a specific marker for mature DC. On the other hand, S100 has been frequently used as a classical marker of both immature and mature DCs [13].

Tumor-infiltrating DCs have been found in the tumor microenvironment in many different types of cancer, including breast, colorectal, melanoma, gastric and esophageal carcinoma [14] [15] [16] [17] [18] [19] [20]. According to these reports, observations of mature dendritic cells at the tumor edge are correlated with a favorable prognosis. However, no information is available regarding the relationship between tumor-infiltrating LAMP-3 DCs and T cells in ESCC.

The aim of this study was to examine the relationship between LAMP-3 DCs and CD8 T cells in tumors in order to clarify the clinical relevance of the local immune microenvironment in ESCC. 


\section{Materials and Methods}

\section{Clinical samples}

Tumor samples were obtained from 80 patients (mean age, 64 years) with primary ESCC who underwent surgical resection without preoperative chemotherapy at the Department of Surgical Oncology, Osaka City University Hospital, between 2003 and 2011. Tumors were diagnosed histologically based on the $11^{\text {th }}$ Edition of the Japanese Classification of Esophageal Cancer [21]. Informed consent was obtained from all patients.

\section{Immunohistochemistry}

Tumor specimens in paraffin-embedded blocks were cut into $4-\mu \mathrm{m}$-thick sections. Nonspecific binding was blocked using nonspecific staining blocking reagent (DAKO, Kyoto, Japan). The sections were then reacted with mouse monoclonal anti-LAMP-3 antibody (clone: 16H11.2 Merck, Darmstadt, Germany), rabbit monoclonal anti-CD8 antibody (clone: EP1150Y; Abcam, Cambridge, UK) and mouse monoclonal anti-S100 antibody (clone: 4C4.9; Abcam, Cambridge, UK) at $4^{\circ} \mathrm{C}$ overnight. Sections were incubated with secondary antibody for $10 \mathrm{~min}$ at room temperature. After washing in phosphate-buffered saline (PBS), the sections were visualized using 3-3'-diaminobenzidine (DAB) for $5 \mathrm{~min}$ and counterstained with hematoxylin. We examined the 
average number of LAMP-3-positive, CD8-positive cells and S100-positive cells in 5 different randomly selected field fields under a light microscope at $\times 400$ magnification.

\section{Immunofluorescence staining of tissue sections}

Tumor specimens in paraffin-embedded blocks were cut into $4-\mu \mathrm{m}$-thick sections. Nonspecific binding was blocked using nonspecific staining blocking reagent (DAKO, Kyoto, Japan). The slides were incubated with a mouse monoclonal anti-LAMP-3 antibody (clone: 16H11.2 Merck, Darmstadt, Germany) and a rabbit monoclonal antiCD8 antibody (clone: EP1150Y; Abcam, Cambridge, UK) at $4{ }^{\circ} \mathrm{C}$ overnight. The slides were subsequently incubated with HiLyte Fluor 488-labeled goat polyclonal anti-mouse IgG antibody (AnaSpec, Fremont, CA, USA) and HiLyte Fluor 647-labeled goat polyclonal anti-rabbit IgG antibody (AnaSpec, Fremont, CA, USA) for 1 hour at room temperature. The sections were covered with glass using DAPI-Fluoromount-G (Southern Biotech). Digital images were taken with an all-in-one fluorescence microscope (BZ8000; Keyence, Osaka, Japan).

Correlation of tumor infiltrating LAMP-3 DCs with CD8 T cells 
We divided the 80 cases into two groups by the median number of LAMP-3 DCs and CD8 T cells to examine the clinicopathological features. With regard to the distribution of CD8 T cells, it has been reported that intratumoral CD8 T cells are the strongest predictor of survival in colorectal cancer [22]. Thus, we next examined the association between the number of infiltrating peritumoral LAMP-3 DCs and the intratumoral CD8 T cells.

\section{Survival Analysis}

The association with overall survival was analyzed initially by a Kaplan-Meier curve and the log-rank test, and also with Cox regression analysis. Overall survival (OS) and disease free survival (DFS) curves were drawn using the Kaplan-Meier method, and a log-lank test was used to assess the significance of differences in the survival. The day of surgery was used as the starting point for the measurement of OS. DFS is defined as the time between the beginning of the treatment and disease progression or death from any cause. Dead cases caused by except esophageal cancer were excluded. A Cox proportional regression model was used for univariate and multivariate analyses of prognostic factors. $P$-values of $<0.05$ were considered to indicate statistical significance. Each statistical analysis was performed using the JMP software program (SAS Institute, Cary, NC, USA). 
Statistical Analysis

Statical analysis was performed using JMP software program (SAS Institute, Cary, NC, USA). The Mann-Whitney test was used to assess the associations between the expression of LAMP-3 and CD8 and clinicopathological features. 


\section{Results}

\section{Patient characteristics}

A total of 80 patients with ESCC were involved in this study. The patients consisted of $64(80 \%)$ males and $16(20 \%)$ females. All patients underwent curative surgery without any preoperative therapy. The median follow-up time was 59 months (range, 5-111 months). Out of these patients, 57 were diagnosed with stage II/III disease and $38(67 \%)$ of these had received postoperative adjuvant chemotherapy. 5-FU/nedaplatin for 26 patients, S-1 for 10 patients, and 5-FU/cisplatin for 2 patients were employed. We analyzed each survival curve, but no significant difference for each regimen were confirmed (data not shown). Of the 80 patients, 27 (34\%) developed recurrent disease. The most common site of recurrence was the lymph nodes (incidence rate, 55.6\%), followed by liver and lung (incidence rate, both $29.6 \%$ ).

The correlation between tumor-infiltrating LAMP-3 DCs/CD8 T cells and the clinicopathological characteristics

On immunohistochemistry, LAMP-3 DCs appeared as irregularly-shaped cells in cancerous stroma. LAMP-3-positive cells were predominantly observed in the peritumoral area and were sparsely observed in the intratumoral area (Fig. 1 A,B,C), 
whereas CD8 T cells were distributed between the peritumoral and intratumoral areas (Fig. 1 D,E,F). Immunofluorescence staining showed clusters of CD8 T cells around LAMP-3 DCs in the peritumoral area (Fig. 1G). As we evaluated the infiltration of the LAMP-3 and CD 8 at the same location in the same specimen, we confirmed the CD8 T cells existed around LAMP-3 DCs in immunohistochemistry and immunofluorescence staining (Fig. 2). The number of infiltrating LAMP-3 DCs and CD8 T cells differed among the regions. The mean number of LAMP-3 DCs infiltrating the peritumoral and intratumoral areas was 9.9 (range, 0 to 24.6) and 0.13 (range, 0 to 2.0), respectively. The mean number of CD8 T cells infiltrating the peritumoral and intratumoral areas was 13.37 (0-42.4) and 5.44 (0-21.6), respectively (Fig. 3A). High peritumoral LAMP-3 DC infiltration was significantly associated with the number of intratumoral CD8 T cells $(p=0.03)$ (Fig. 3B). We observed that the infiltration number of LAMP-3 DCs and CD8 $\mathrm{T}$ cells had a weak positive correlation ( $r=0.24, p=0.033)$ (Fig. 3C). Immunohistochemistry staining showed that S100 DCs were found in both the peritumoral and intratumoral areas (Fig. 4A) as contrast to LAMP-3 DCs (Fig. 4B). We detected no significant correlation between the number of infiltration of LAMP-3 DCs and S100 DCs $(r=0.15, p=0.25)$ (Fig. 4C). 
The association between the number of tumor-infiltrating LAMP-3 DCs or CD8 T cells and the clinicopathological features is shown in Table 1. The peritumoral infiltration of LAMP-3 DCs was significantly decreased according to the extent of the primary tumor (pT), lymph node metastasis ( $\mathrm{pN}$ ), tumor, node, metastasis stage (pStage), and positive lymphatic invasion (Ly). The intratumoral infiltration of CD8 T cells was associated with lymphatic invasion. The most invasive tumor area of the whole tissue slide was selected, and we evaluated the dominant components of the stroma by hematoxylin-eosin staining. We classified the stromal type into two types (Fig 2E a: lymphocyte dominant type b: fibroblast dominant type). We scored the components to the nearest $10 \%$, and the sample was classified by the predominant components. To clarify the effect of stromal tissue, we evaluated the correlation with the stromal type by hematoxylin-eosin staining (Fig. 5). Stromal type was not the significant correlation factor with LAMP-3 DCs or CD8 T cells (Table 1).

The prognostic significance of tumor-infiltrating LAMP-3 DCs and CD8 T cells We evaluated the prognostic impact of tumor-infiltrating LAMP-3 DCs and CD8 T cells in the primary tumor. A univariate analysis revealed that low infiltration by LAMP-3 DCs and CD8 $\mathrm{T}$ cells was associated with a poor prognosis, similar to $\mathrm{pT}, \mathrm{pN}$, pStage and 
lymphatic invasion (Ly). In a multivariate analysis, $\mathrm{pT}, \mathrm{pN}$ and peritumoral/ intratumoral CD8 T cells were independently associated with OS (Table 2). Patients with low peritumoral LAMP-3 DC infiltration had a significantly poorer prognosis than those with high infiltration (Fig. 6A). The 5-year survival rate was $81 \%$ and $57 \%$ for patients with high and low LAMP-3 infiltration, respectively. With regard to intratumoral CD8 T cells, patients with low intratumoral CD8 $\mathrm{T}$ cell infiltration had a worse prognosis than those with high infiltration (Fig. 6B). Patients with both high LAMP-3 and high CD8 infiltration had a significantly better prognosis than those with any other degree of LAMP-3 and CD8 of infiltration (Fig. 6C). We divided the patients into high or low groups according to the $\mathrm{T}$ factor or pathological stage. Although there was no significant difference, the high infiltration groups of LAMP-3 DC had a better prognosis than the low infiltration groups in each analyze (Fig. 7). The 5-year survival rate was $95 \%$ and $86 \%$ for patients with high and low LAMP-3 DCs infiltration in the T1 group, $65 \%$ and $43 \%$ in the T2 group. On the other hand, the 5-year survival rate was $96 \%$ and $83 \%$ for patients with high and low LAMP-3 DCs infiltration in the pStage 0 -II group, $50 \%$ and $38 \%$ in the pStage III group. 


\section{Discussion}

In this study, we showed that tumor-infiltrating LAMP-3 DCs were predominantly present in the peritumoral region accompanied by CD8 $\mathrm{T}$ cells and were associated with a favorable prognosis in ESCC.

A number of studies have found associations between the presence of infiltrating immune cells in the tumor microenvironment and the prognosis. It has been reported that the high infiltration of T cells (namely TILs), including CD8 ${ }^{+}$cytotoxic T lymphocytes (CTLs), was associated with a favorable prognosis in cancer patients, including those with esophageal cancer [3]. Furthermore, a high degree of mature DC infiltration has been reported to be associated with a favorable prognosis in many types of cancer, including melanoma and breast cancer [14] [23]. Consistent with these reports, our study showed that the prognosis of patients with high degree of TIL and LAMP3 DC infiltration was obviously better than that of patients with low degrees of infiltration. Furthermore, we detected a positive correlation between the number of peritumoral LAMP-3 DCs and the number of intratumoral CD8 $\mathrm{T}$ cells. On the other hand, there was no significant correlation between the number of infiltration of LAMP-3 DCs and S100 DCs, indicating that the LAMP-3 DCs might be " mature" DCs. LAMP-3 DC infiltration was not an independent prognostic factor according to a multivariate analysis, likely because of the 
strong confounding effects that LAMP-3 DCs and intratumoral CD8 T cells had on each other.

DCs play an integral role in influencing the immune system to induce the production of antitumor CTLs; however, their role may change from immunostimulatory to immunosuppressive, and they may produce tumor-associated macrophages and myeloidderived suppressor cells [24]. Not surprisingly, DC infiltration into tumors has been reported to affect the prognosis both positively and negatively [13]. To induce CTLs, "mature" DCs should migrate into the lymph nodes, where they prime CTLs, further augmenting the anti-tumor immune response [25]. The phenotypes of mature DCs include enhanced MHCs and co-stimulatory molecules, such as CD83 and LAMP-3. The correlation of the LAMP-3 expression with DC maturation has been directly established by in vitro studies[11] [12]. LAMP-3 plays an important role during the presentation process of exogeneous antigens to T cells. Thus, LAMP-3-expressing DCs may represent "bona fide" mature DCs that induce an anti-tumor immune response. In the present study, we focused on LAMP-3-expressing mature DCs in the tumor microenvironment. In addition to the effects of their maturation state on the prognosis, the distribution of tumor-infiltrating DCs may also influence the prognosis. Several studies have shown that immature DCs expressing CD1a are located throughout tumor, whereas LAMP-3 mature 
DCs reside in the peritumoral areas [14] [15] [16] [17] [19]. Consistent with these reports, we showed that LAMP-3 DCs were predominantly located in the peritumoral area. We also observed the aggregation of CD8 T cells around LAMP-3 DCs, resulting in the formation of LAMP-3 DC-CD8 T cell clusters. It is reported that LAMP-3 is expressed particularly strongly on the interdigitating dendritic cells located in the T cell areas of the lymph nodes [11], and that T cells clustered around mature DCs can induce the activation of T cells to promote anti-tumor immunity [14] [16] [26]. The density values of LAMP3 DCs and activated $\mathrm{CD} 25^{+}$and $\mathrm{OX} 40^{+}$lymphocytes in the tumor site have been reported to be strongly correlated, and these are independent prognostic factors in melanoma [14]. In addition, $\mathrm{T}$ cells have been reported to be clustered around mature DCs in the peritumoral areas, and the majority of infiltrating $\mathrm{T}$ cells in breast carcinoma (70-75\%) were found to be CD8 T cells, with a fraction displaying CD69 and CD25, markers of early activation [16]. Furthermore, mature DC-lymphocyte clusters have been observed in the cancer stroma at the invasive margin in colorectal cancer, with clusters composed largely of CD4 T cells and containing a small number of CD8 T cells and CD20 B cells [26]. Importantly, we showed the existence of CD8 T cells around LAMP-3 DCs, indicating that LAMP-3 DCs present tumor-antigens to $\mathrm{T}$ cells in the tumor microenvironment of ESCC. 
It has been reported that stromal type was significant prognostic factor in breast cancer [27]. In this study, we could not observe significant correlation between stroma type and tumor infiltrating LAMP-3 DCs or CD8 T cells in ESCC.

The present study is associated with several limitations. We did not demonstrate how tumor-infiltrating DCs interact with tumor cells and other immune cells in the local tumor area. The migration of DCs might be related to the coordinated action of chemokines [28]. Tumor-infiltrating DCs might reflect the local immune response to signals provided by the tumor microenvironment. Further investigations will be required to understand the precise mechanism underlying DC migration at tumor sites and the induction of CD8 T cells by LAMP-3 DCs in the tumor microenvironment. In addition, more detailed studies will be needed to explore the functional aspects of peritumoral LAMP-3 DCs.

In the present study, we observed the presence of mature LAMP-3 DCs with CD8 T cells in the peritumoral region, and the high infiltration of LAMP-3 DCs was significantly associated with a favorable prognosis among patients with ESCC. Our results suggest that the anti-tumor immune response might be induced by mature DCs that are located at the local tumor site in ESCC. 


\section{Conclusion}

In conclusion, mature DCs in the tumor region have the strong potential to induce an antitumor immune response. Our findings suggest that tumor-infiltrating LAMP-3 DCs may be a sign of a functional immune response associated with a favorable outcome in patients with ESCC.

\section{Compliance with ethical standards}

Ethical statement

Our investigation was carried out according to the Declaration of Helsinki. All experimental procedures after 2013 were approved as No. 3138 by the Osaka City University ethics committee, and all patients had provided informed consent for collection and analysis of the specimens. On the other hand, because the other experimental procedures up to 2013 were only all-inclusive consent, we are showing optout form as an observational study.

Conflict of interest

All authors declare that there is no conflict of interest in the present study. 


\section{Reference}

1. Ohashi S, Miyamoto S, Kikuchi O, et al. Recent Advances From Basic and Clinical Studies of Esophageal Squamous Cell Carcinoma. Gastroenterology. 2015;149:170015.

2. Kojima T, Doi T. Immunotherapy for Esophageal Squamous Cell Carcinoma. Curr Oncol Rep. 2017;19:017-0590.

3. Fridman WH, Pages F, Sautes-Fridman C, et al. The immune contexture in human tumours: impact on clinical outcome. Nat Rev Cancer. 2012 Mar 15;12(4):298-306. doi: $10.1038 / \mathrm{nrc} 3245$.

4. Zheng X, Song X, Shao Y, et al. Prognostic Role of Tumor-Infiltrating Lymphocytes in Esophagus Cancer: a Meta-Analysis. Cell Physiol Biochem. 2018;45:720-32.

5. Sudo T, Nishida R, Kawahara A, et al. Clinical Impact of Tumor-Infiltrating Lymphocytes in Esophageal Squamous Cell Carcinoma. Ann Surg Oncol. 2017;24:3763-70.

6. Li J, Tang Y, Huang L, et al. A high number of stromal tumor-infiltrating lymphocytes is a favorable independent prognostic factor in M0 (stages I-III) esophageal squamous cell carcinoma. Dis Esophagus. 2017;30:1-7.

7. Lill PH, Fortner GW. Identification and cytotoxic reactivity of inflammatory cells recovered from progressing or regressing syngeneic UV-induced murine tumors. J Immunol. 1978;121:1854-60.

8. Pepper M, Jenkins MK. Origins of CD4(+) effector and central memory T cells. Nat Immunol. 2011;12:467-71.

9. Mildner A, Jung S. Development and function of dendritic cell subsets. Immunity. 2014;40:642-56.

10. Gardner A, Ruffell B. Dendritic Cells and Cancer Immunity. Trends Immunol. 2016;37:855-65.

11. de Saint-Vis B, Vincent J, Vandenabeele S, et al. A novel lysosome-associated membrane glycoprotein, DC-LAMP, induced upon DC maturation, is transiently expressed in MHC class II compartment. Immunity. 1998;9:325-36.

12. Barois N, de Saint-Vis B, Lebecque S, et al. MHC class II compartments in human dendritic cells undergo profound structural changes upon activation. Traffic. 2002;3:894-905.

13. Karthaus N, Torensma R, Tel J. Deciphering the message broadcast by tumorinfiltrating dendritic cells. Am J Pathol. 2012;181:733-42.

14. Ladanyi A, Kiss J, Somlai B, et al. Density of DC-LAMP(+) mature dendritic cells in combination with activated T lymphocytes infiltrating primary cutaneous 
melanoma is a strong independent prognostic factor. Cancer Immunol Immunother. 2007:56:1459-69.

15. Sandel MH, Dadabayev AR, Menon AG, et al. Prognostic value of tumor-infiltrating dendritic cells in colorectal cancer: role of maturation status and intratumoral localization. Clin Cancer Res. 2005;11:2576-82.

16. Bell D, Chomarat P, Broyles D, et al. In breast carcinoma tissue, immature dendritic cells reside within the tumor, whereas mature dendritic cells are located in peritumoral areas. J Exp Med. 1999;190:1417-26.

17. Treilleux I, Blay JY, Bendriss-Vermare N, et al. Dendritic cell infiltration and prognosis of early stage breast cancer. Clin Cancer Res. 2004;10:7466-74.

18. Nagorsen D, Voigt S, Berg E, et al. Tumor-infiltrating macrophages and dendritic cells in human colorectal cancer: relation to local regulatory T cells, systemic T-cell response against tumor-associated antigens and survival. J Transl Med. 2007;5:1479-5876.

19. Liu J, Lu G, Li Z, et al. Distinct compartmental distribution of mature and immature dendritic cells in esophageal squamous cell carcinoma. Pathol Res Pract. 2010;206:602-6.

20. Ishigami S, Ueno S, Matsumoto M, et al. Prognostic value of CD208-positive cell infiltration in gastric cancer. Cancer Immunol Immunother. 2010;59:389-95.

21. Japan Esophageal S. Japanese Classification of Esophageal Cancer, 11th Edition: part I. Esophagus. 2017;14:1-36.

22. Richards CH, Roxburgh CS, Powell AG, et al. The clinical utility of the local inflammatory response in colorectal cancer. Eur J Cancer. 2014;50:309-19.

23. Iwamoto M, Shinohara H, Miyamoto A, et al. Prognostic value of tumor-infiltrating dendritic cells expressing CD83 in human breast carcinomas. Int J Cancer. 2003;104:92-7.

24. Shurin GV, Ma Y, Shurin MR. Immunosuppressive Mechanisms of Regulatory Dendritic Cells in Cancer. Cancer Microenvironment. 2013;6:159-67.

25. Mempel TR, Henrickson SE, Von Andrian UH. T-cell priming by dendritic cells in lymph nodes occurs in three distinct phases. Nature. 2004;427:154-9.

26. Suzuki A, Masuda A, Nagata H, et al. Mature dendritic cells make clusters with T cells in the invasive margin of colorectal carcinoma. J Pathol. 2002;196:37-43.

27. Ahn S, Cho J, Sung J, et al. The prognostic significance of tumor-associated stroma in invasive breast carcinoma. Tumour Biol. 2012;33:1573-80.

28. Johnson LA, Jackson DG. Control of dendritic cell trafficking in lymphatics by chemokines. Angiogenesis. 2014;17:335-45. 


\section{Figure Legends}

\section{Figure 1}

(A) Immunohistochemical staining of LAMP-3 DCs and CD8 $\mathrm{T}$ cells in ESCC. Microscopy of typical LAMP-3 and CD8 immunohistochemical staining of tumor specimens from a patient with ESCC. The membrane and cytoplasm of LAMP-3 DCs showed dark-brown staining $(\times 400)$. (B) (C) LAMP-3 DCs were mainly distributed in the peritumoral area but were sparsely present in the intratumoral area (B: high infiltration, C: low infiltration $\times 200$ ). (D) (E) (F) CD8 T cells were found in both the peritumoral and intratumoral areas $(\mathrm{D} \times 400, \mathrm{E}$ : high infiltration, F: low infiltration $\times 200)$. $(\mathrm{G})$ Immunofluorescence staining of LAMP-3 DCs and CD8 T cells in the primary tumor of patients with ESCC. Nuclei of cells in primary tumors were stained with DAPI (a), mature DCs stained with anti-LAMP-3 antibody (green) (b), CD8 T cells stained anti-CD8antibody (red) (c) and a merged image (d) are shown. Clusters of CD8 T cells around LAMP-3 DCs can be seen in the peritumoral area (white arrow) 
Fig.1
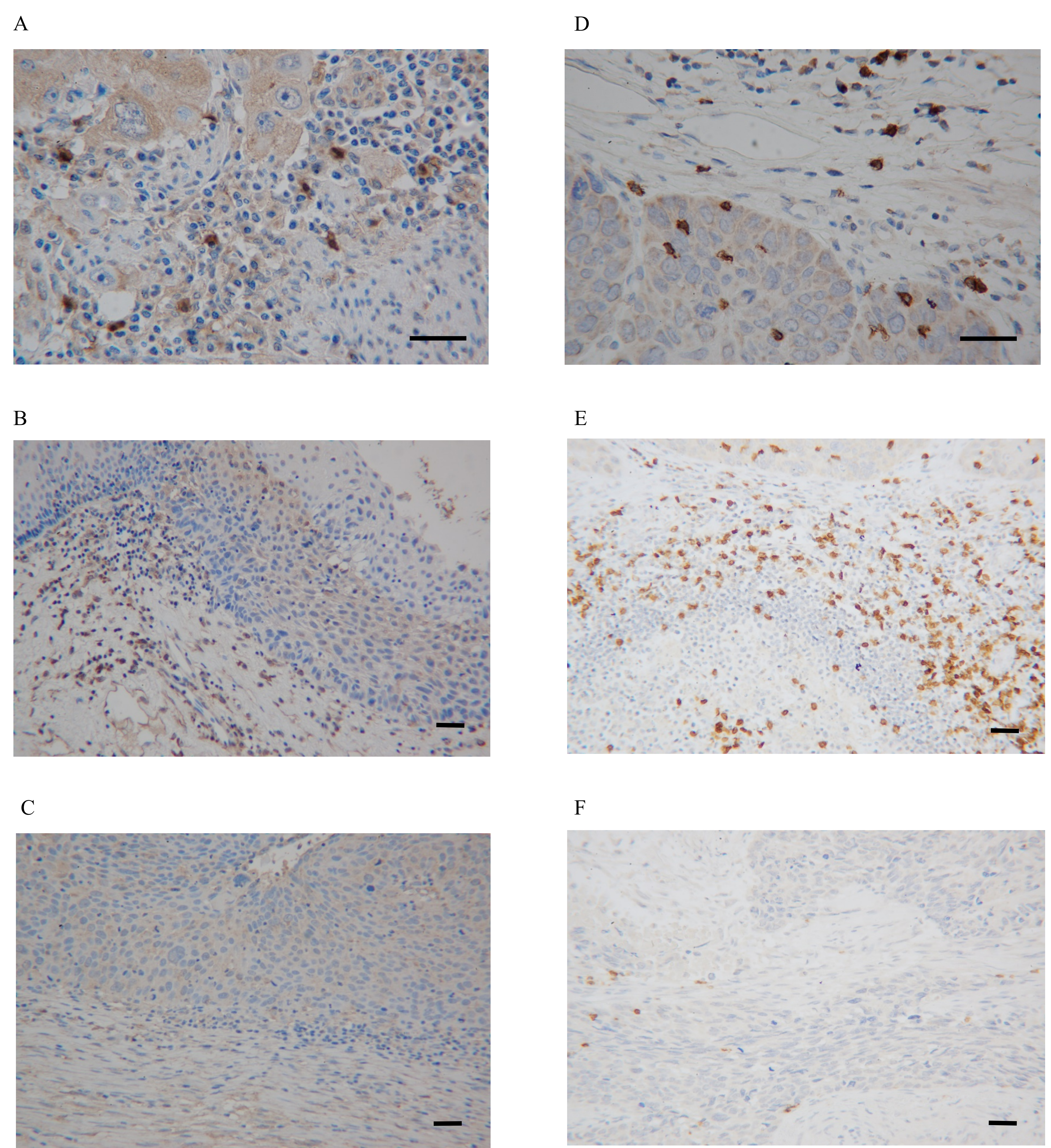

G

(a)

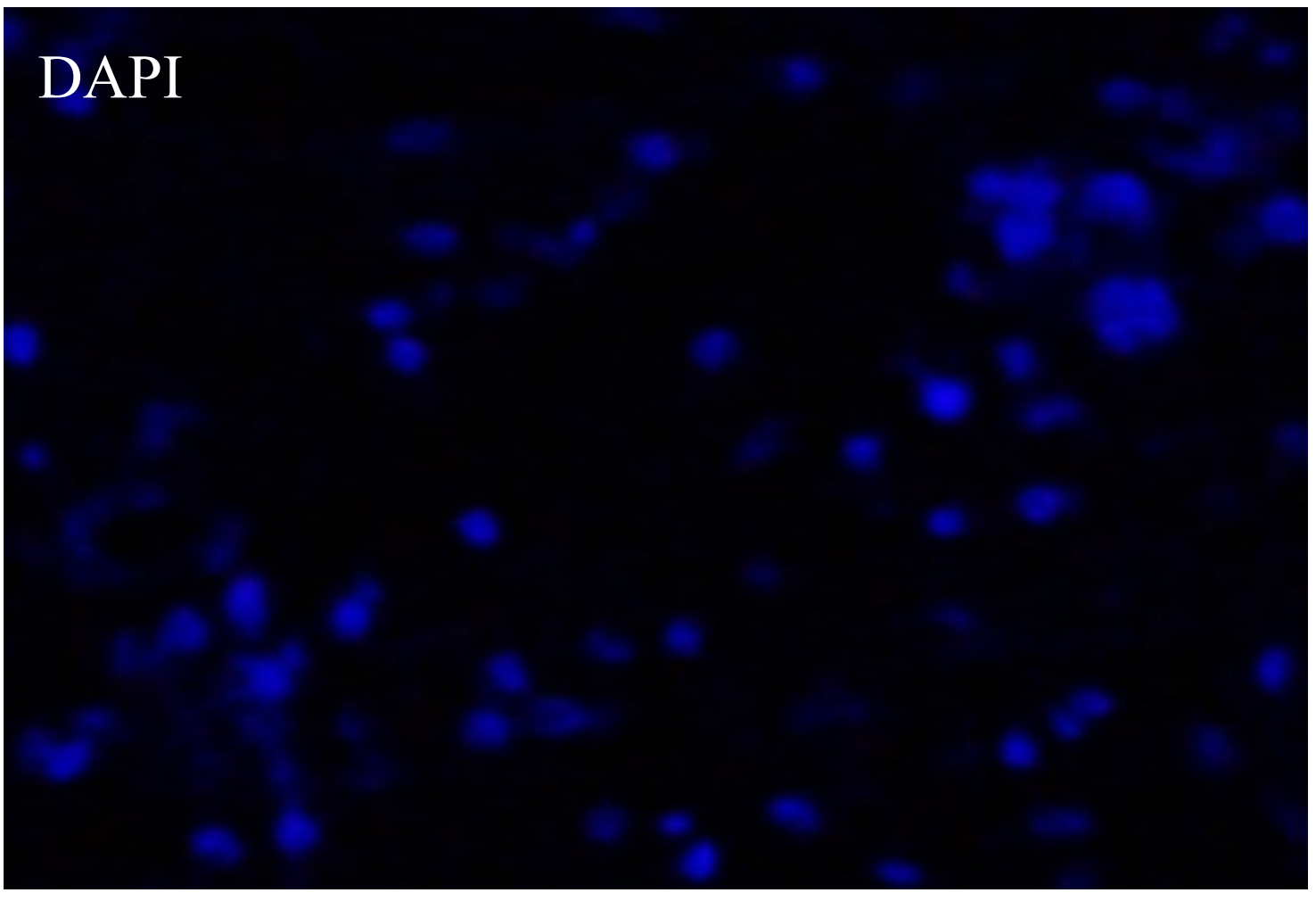

(b)

(c)

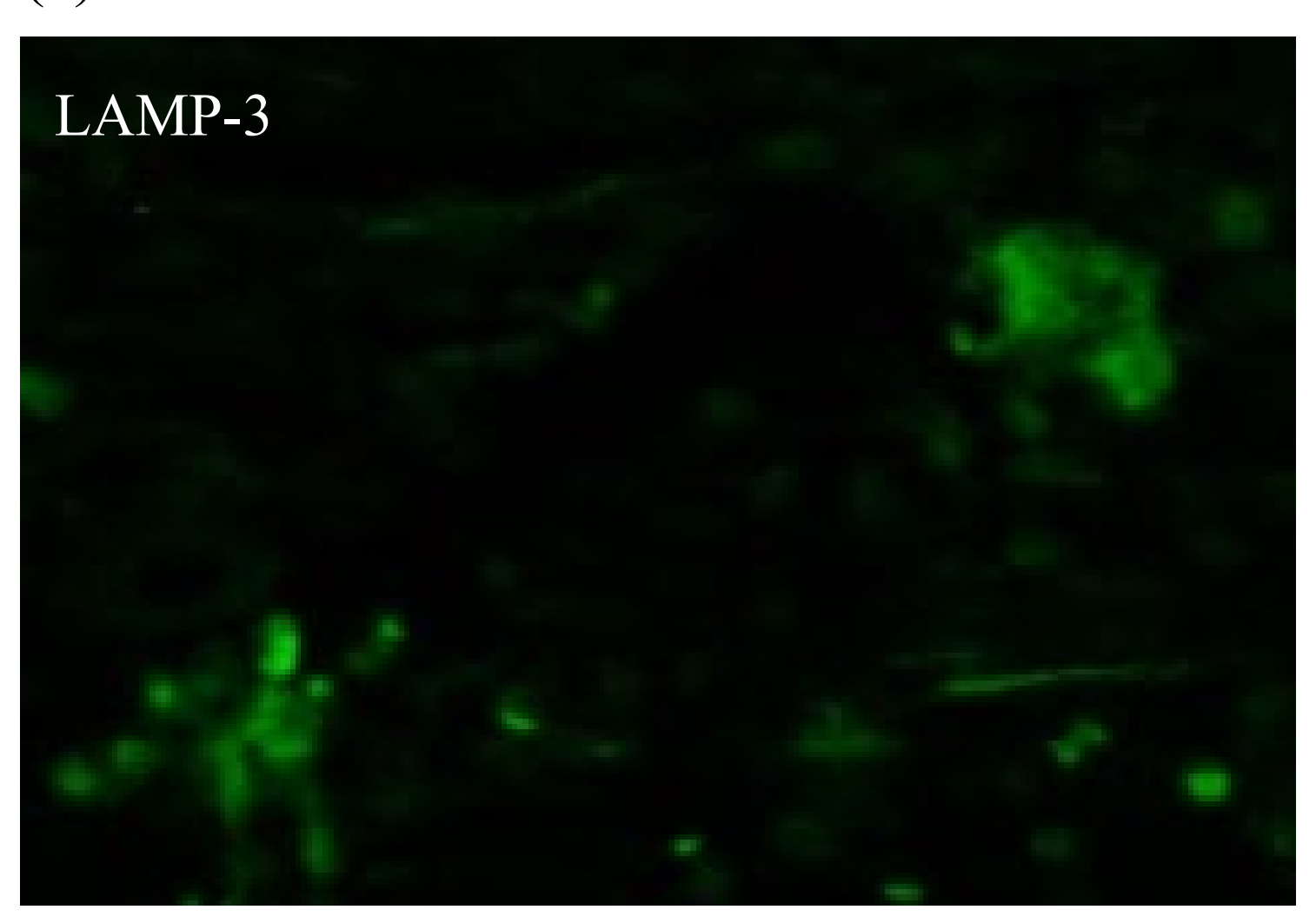

(d)

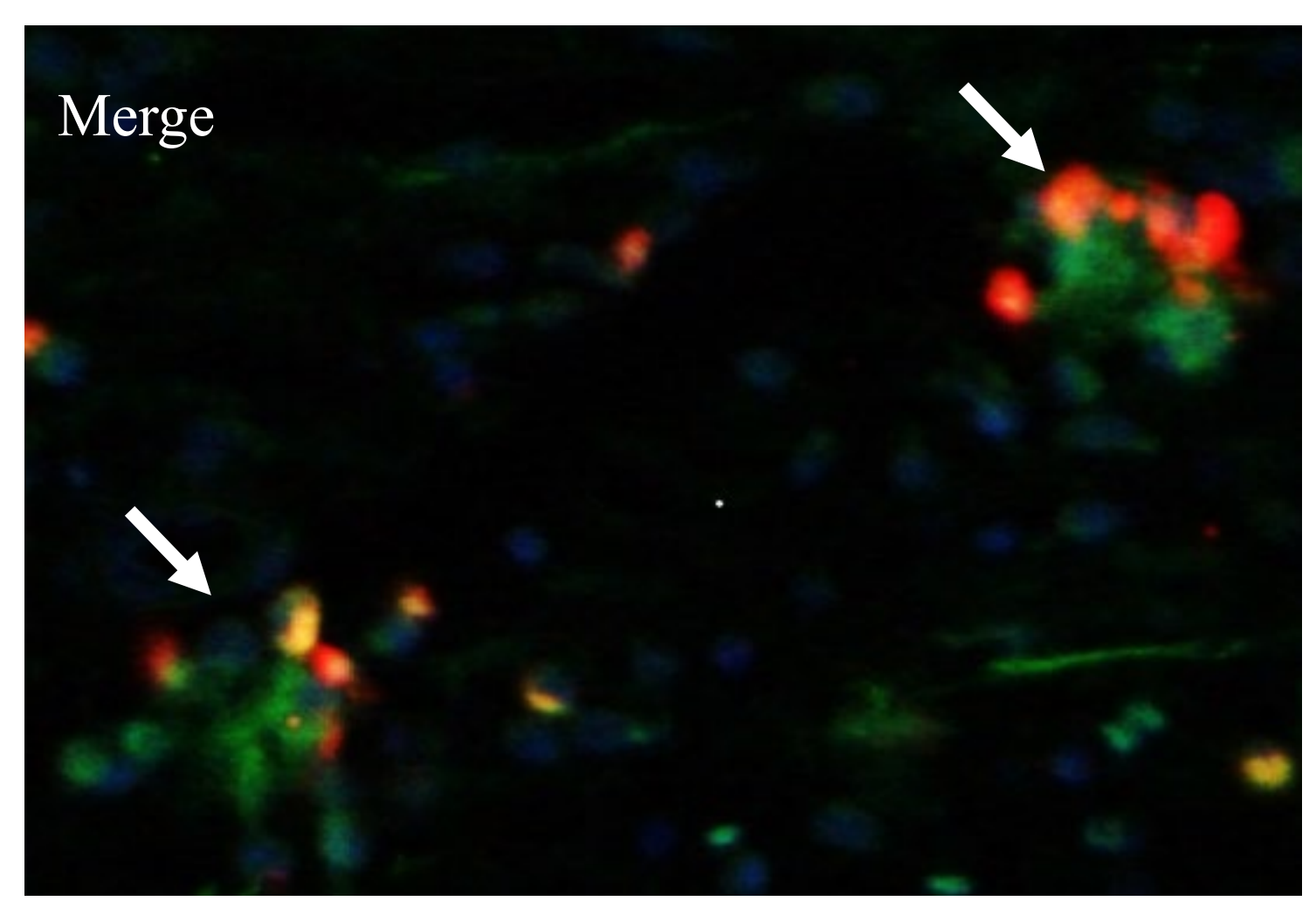




\section{Figure 2}

Immunohistochemical staining of LAMP-3 DCs, CD8 T cells in ESCC at the same location in the same specimen (A: LAMP-3; (a) $\times 200$, (b) $\times 400$, B: CD-8; (a) $\times 200$, (b) $\times 400$ ). (C) Immunofluorescence staining of LAMP-3 DCs and CD8 T cells in the primary tumor of patients with ESCC. Nuclei of cells in primary tumors were stained with DAPI (a), mature DCs stained with anti-LAMP-3 antibody (green, white arrow) (b), CD8 T cells stained anti-CD8-antibody (red, white arrow) (c) and a merged image (d) are shown. Clusters of CD8 T cells around LAMP-3 DCs can be seen in the peritumoral area (white arrow). 
Fig.2

(a)

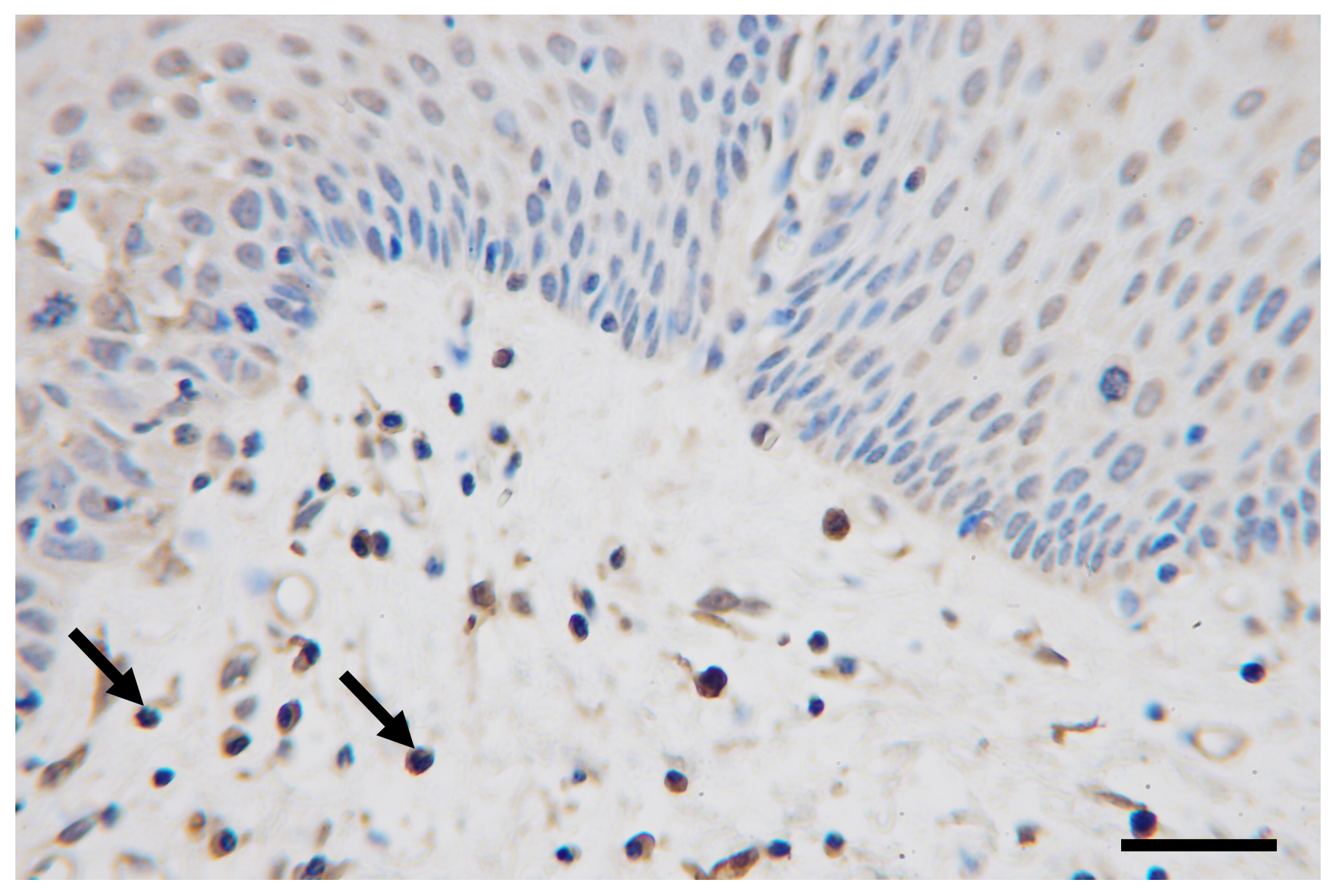

(b)

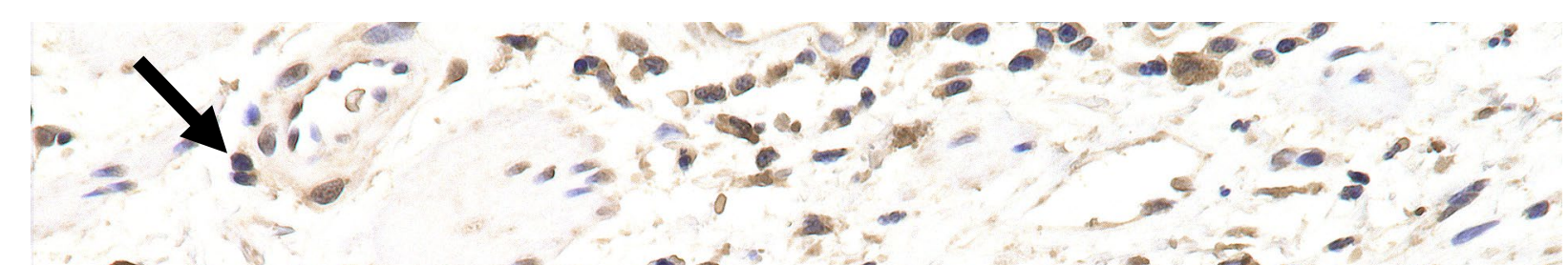

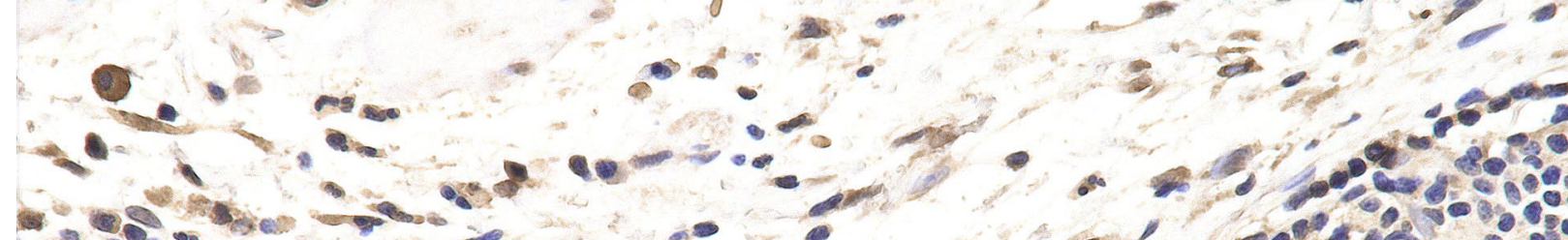

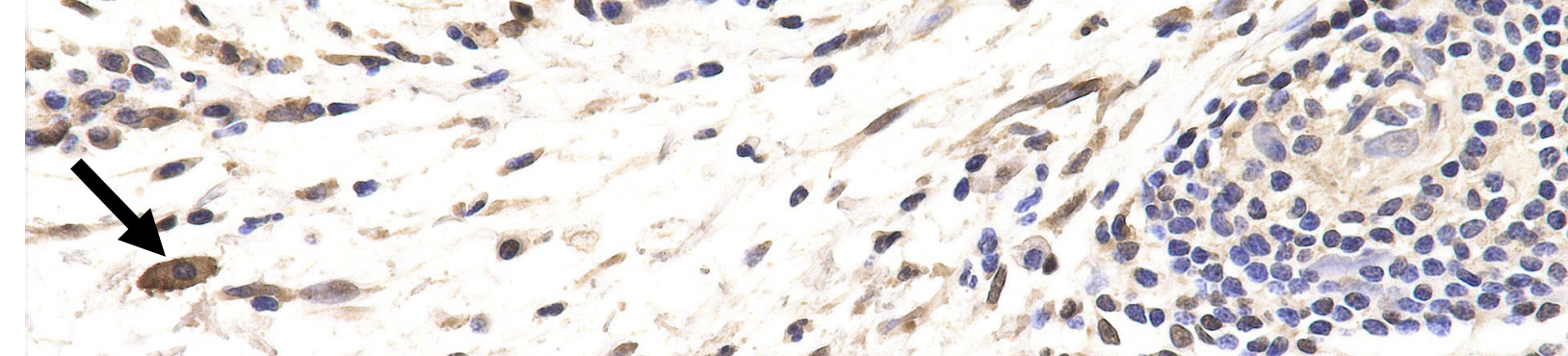

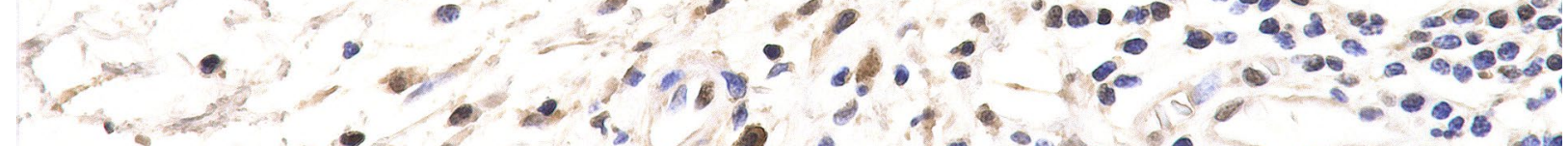

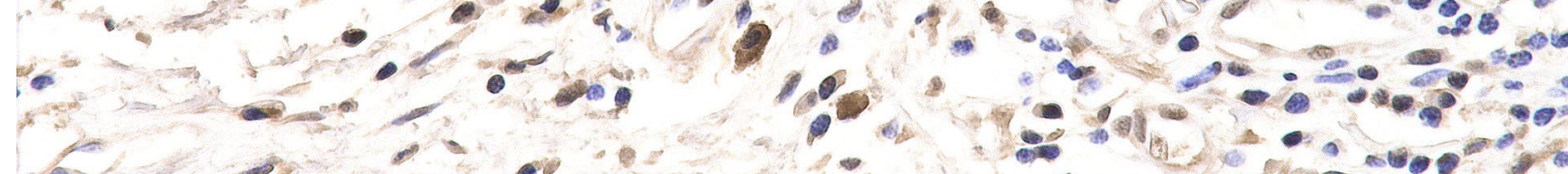

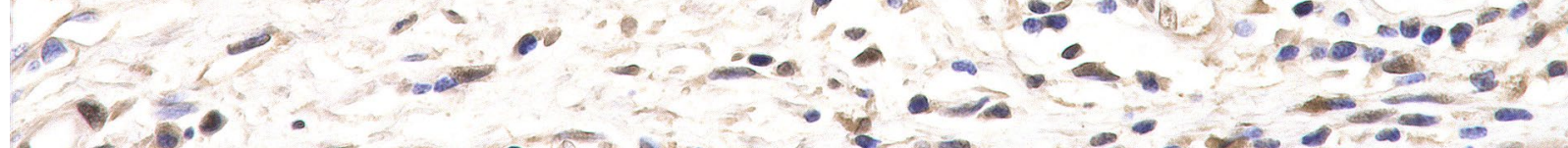

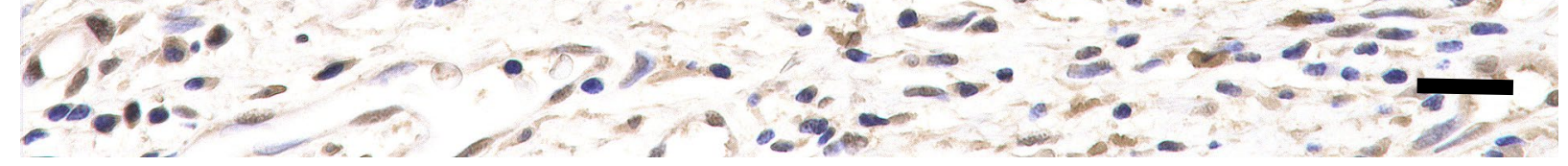

(a)

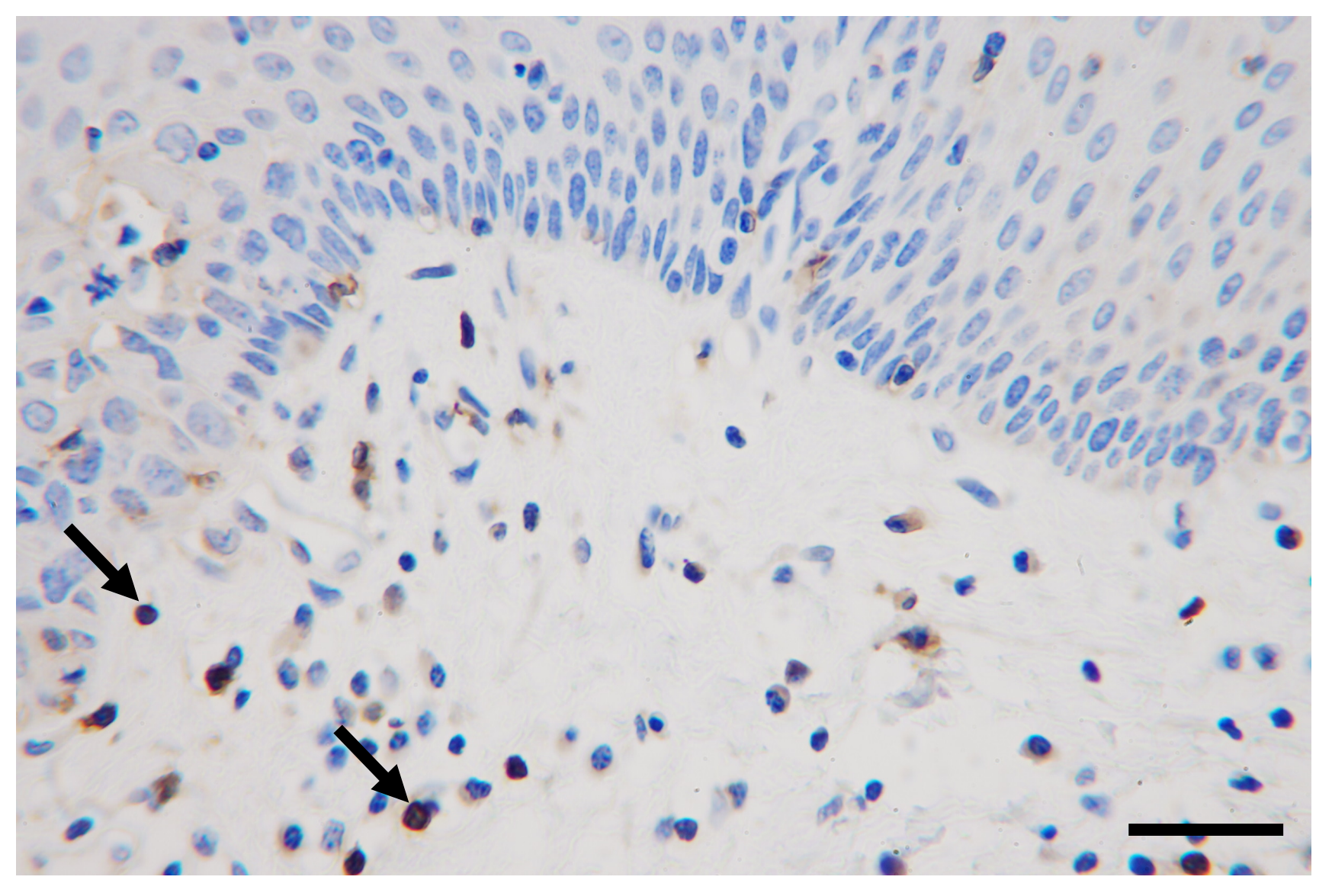

(b)

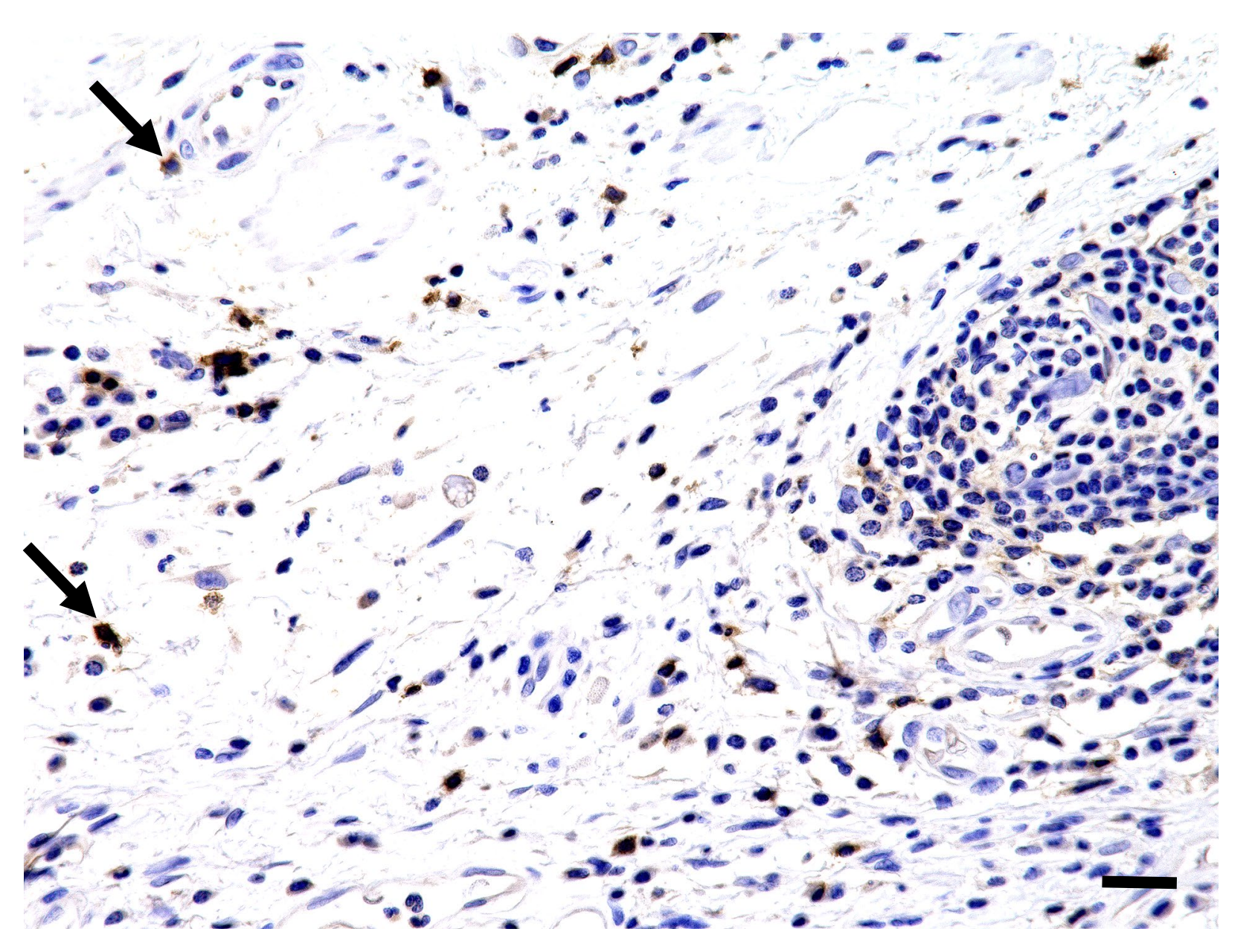

\section{DAPI}

(b) LAMP-3

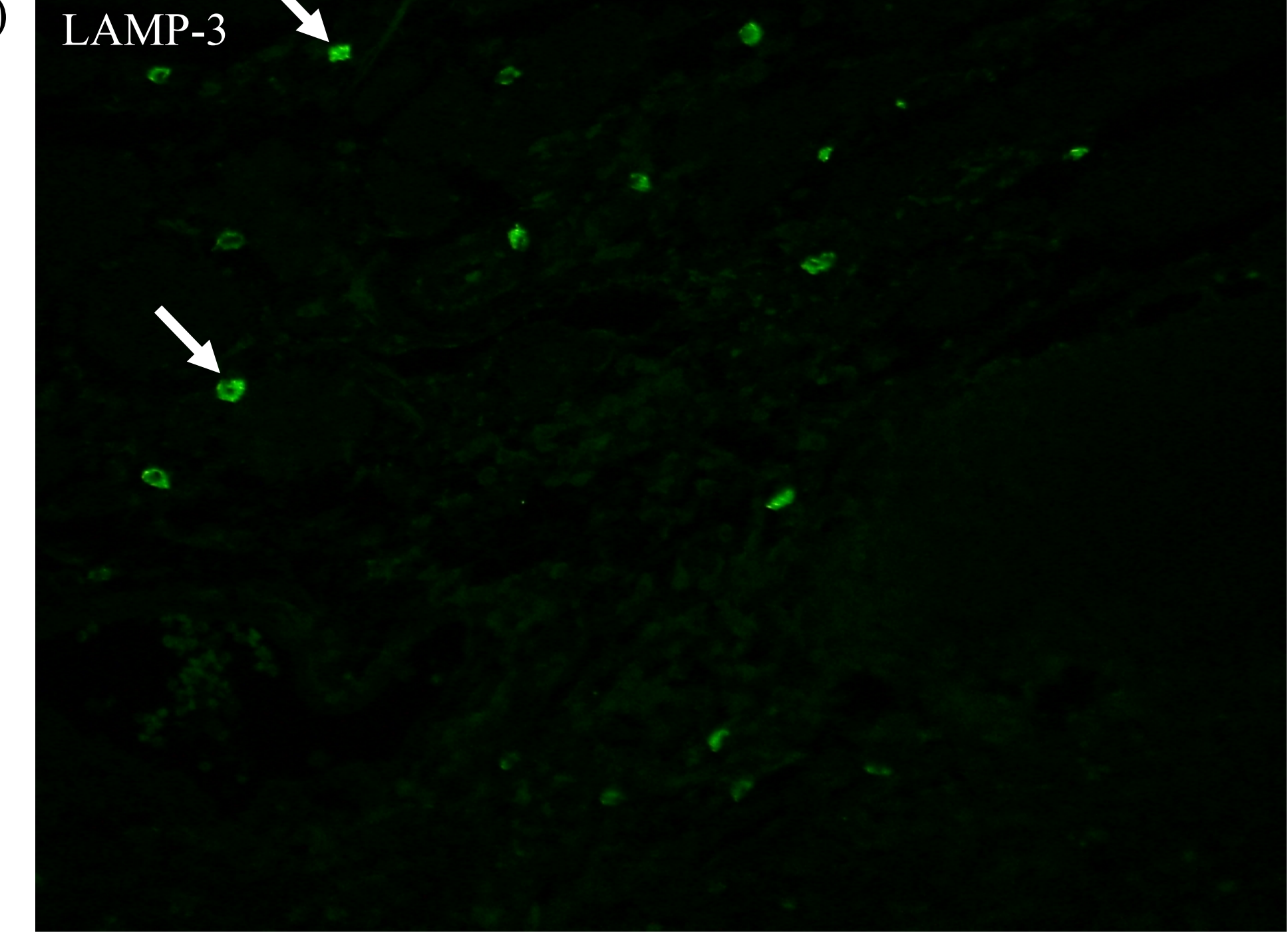

(c)
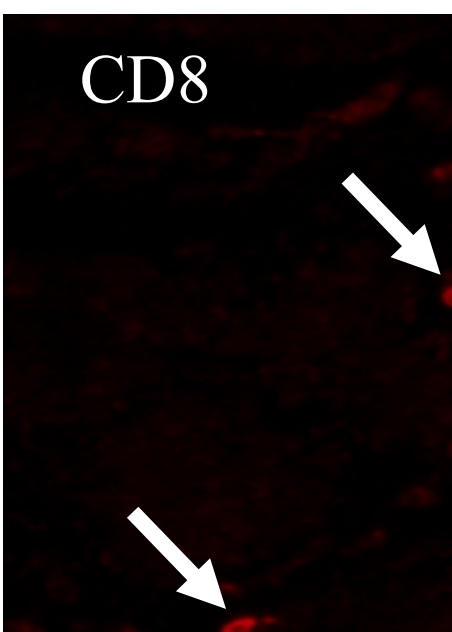

(d)

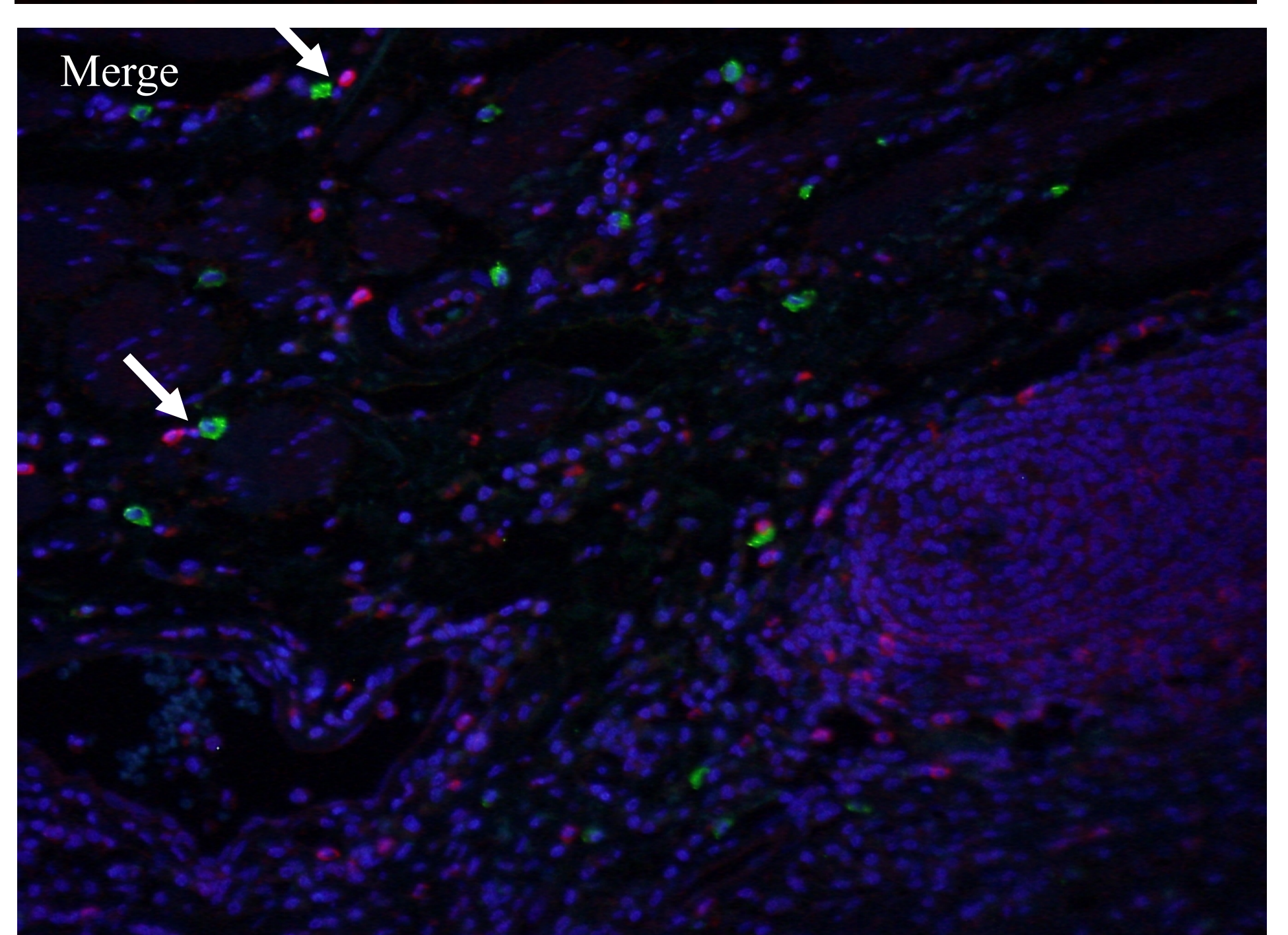




\section{Figure 3}

(A) The mean respective numbers of tumor-infiltrating LAMP-3 DCs and CD8 T cells are shown by location. (B) A comparison of the density of CD8 T cells in locations with a low or high density of LAMP-3 DCs $(p=0.03)$. These groups were divided according to the median number of tumor-infiltrating LAMP-3 DCs. (C) Spearman's correlation coefficients were used to determine the relationship between the number of peritumoralLAMP-3 DCs and the number of intratumoral- CD8 T cells. The number of intratumoralCD8 T cells had a significantly positive correlation with the number of peritumoralLAMP-3 DCs $(r=0.24, p=0.033)$. 
Fig. 3

A.
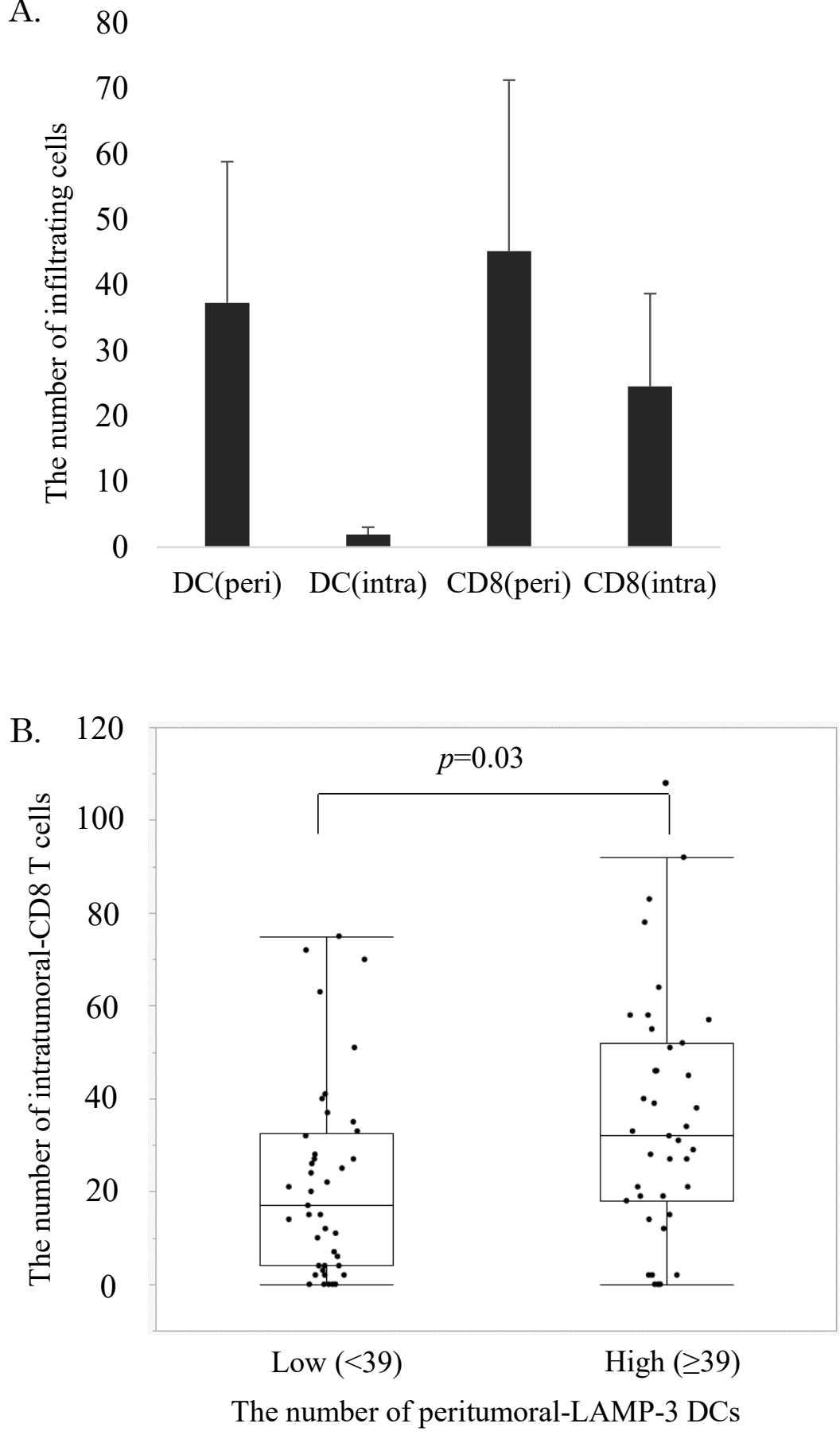

C.

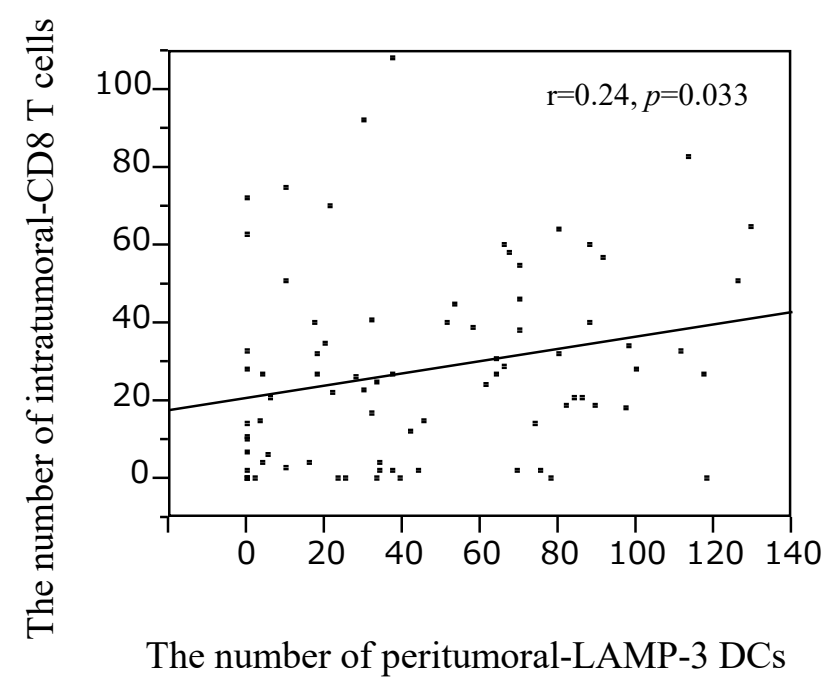




\section{Figure 4}

Immunohistochemical staining of S100 DCs and LAMP-3 DCs in ESCC.

S100 DCs were found in both the peritumoral and intratumoral areas as contrast to

LAMP-3 DCs (A: S100; (a) ×200, (b) ×400, black arrow, B: LAMP-3; (a) ×200, (b) ×400,

black arrow). (C) There is no correlation between the number of S100 DCs and LAMP-3

$\operatorname{DCs}(r=0.15, p=0.25)$. 
Fig.4

A

(a)

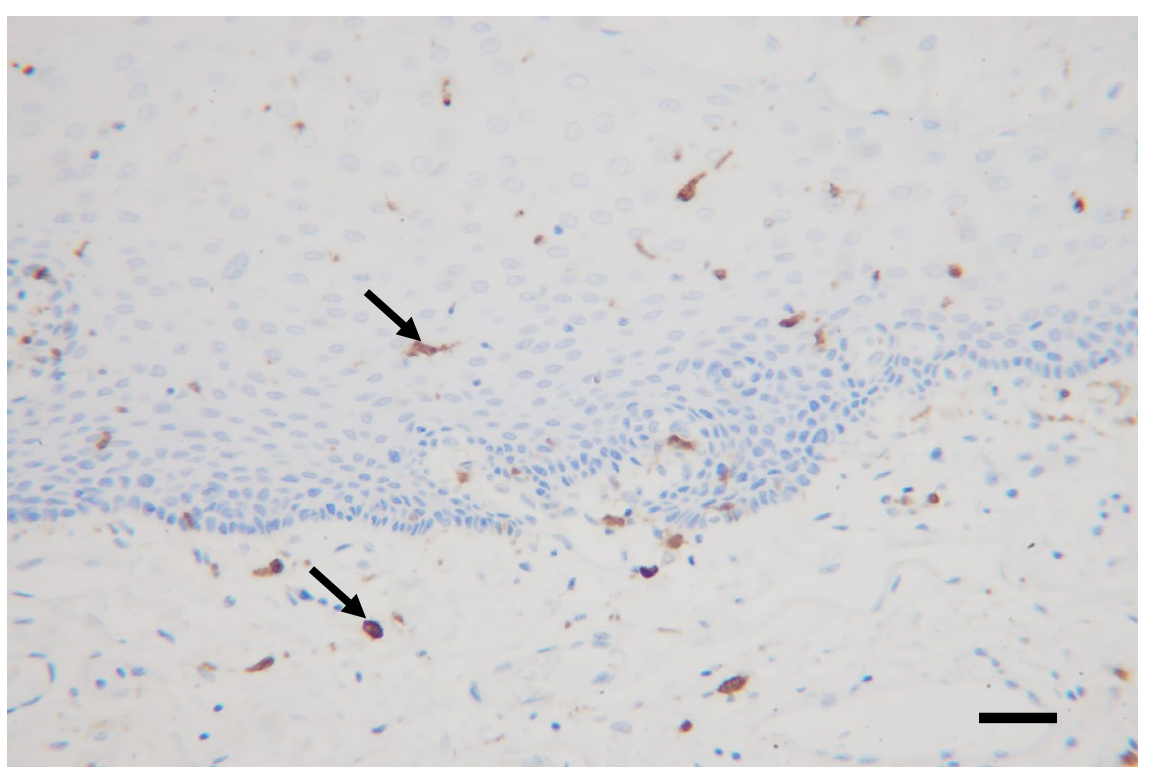

(b)

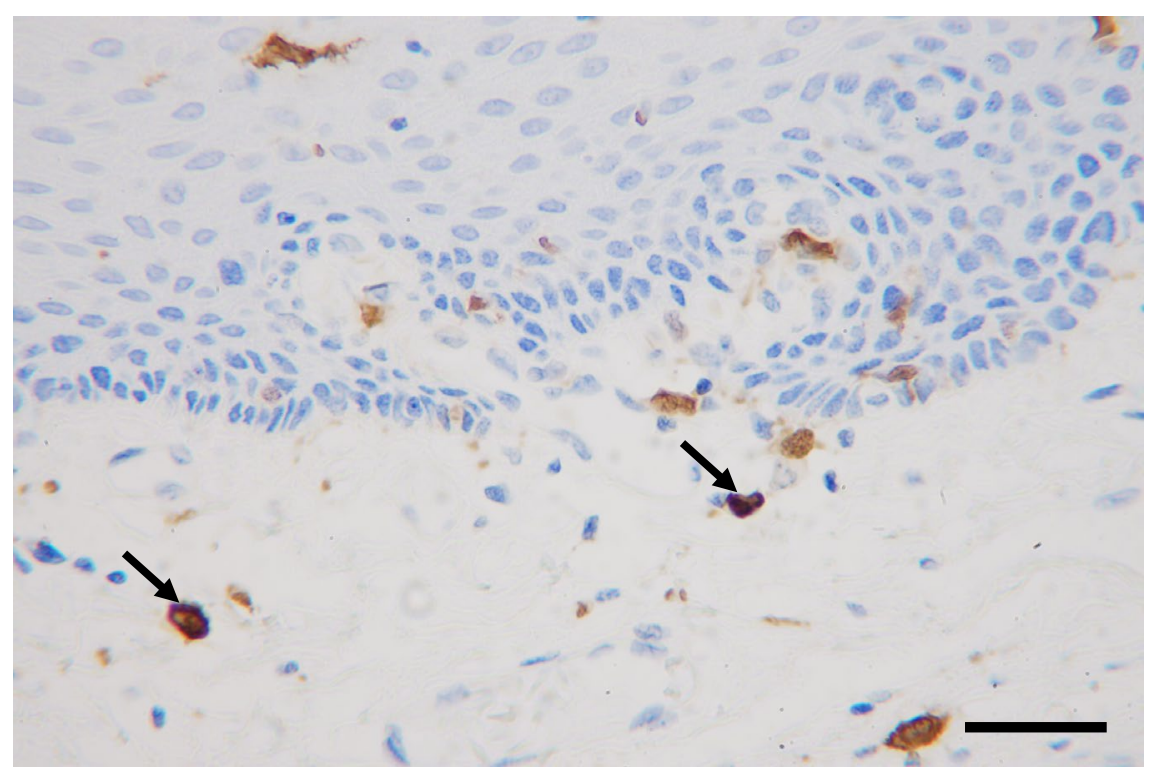

B

(a)

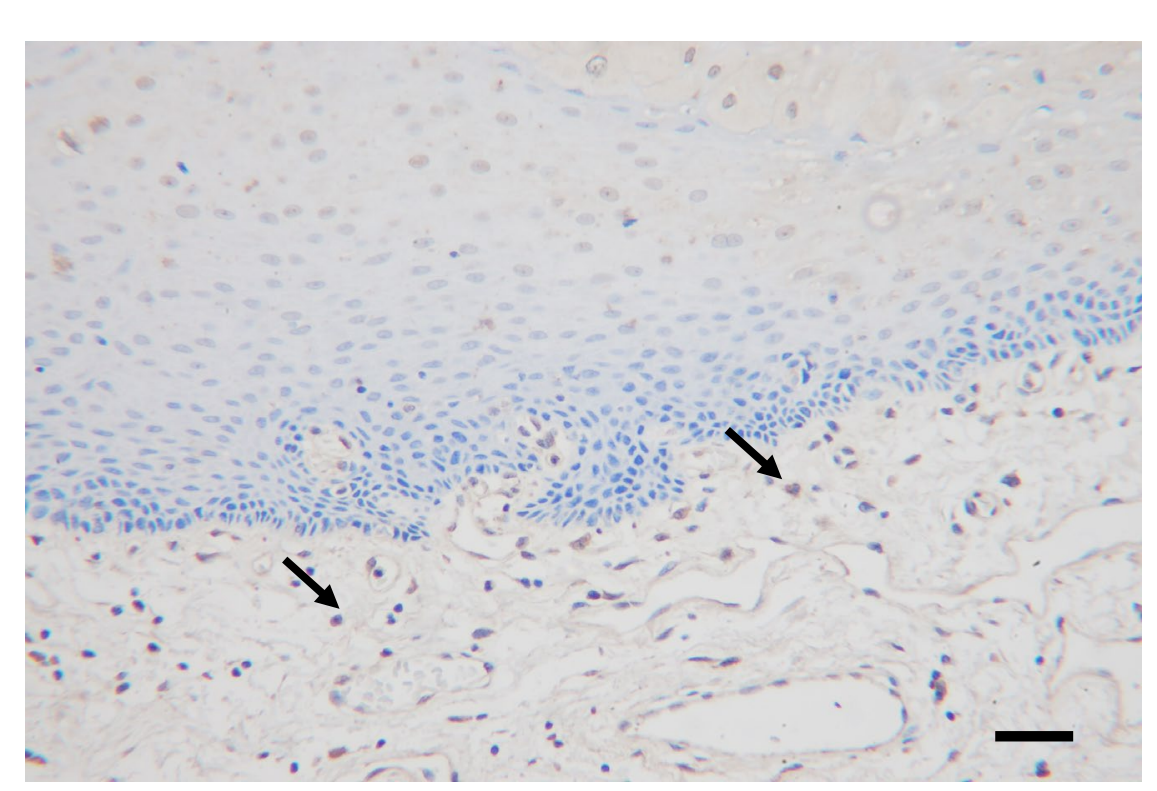

(b)

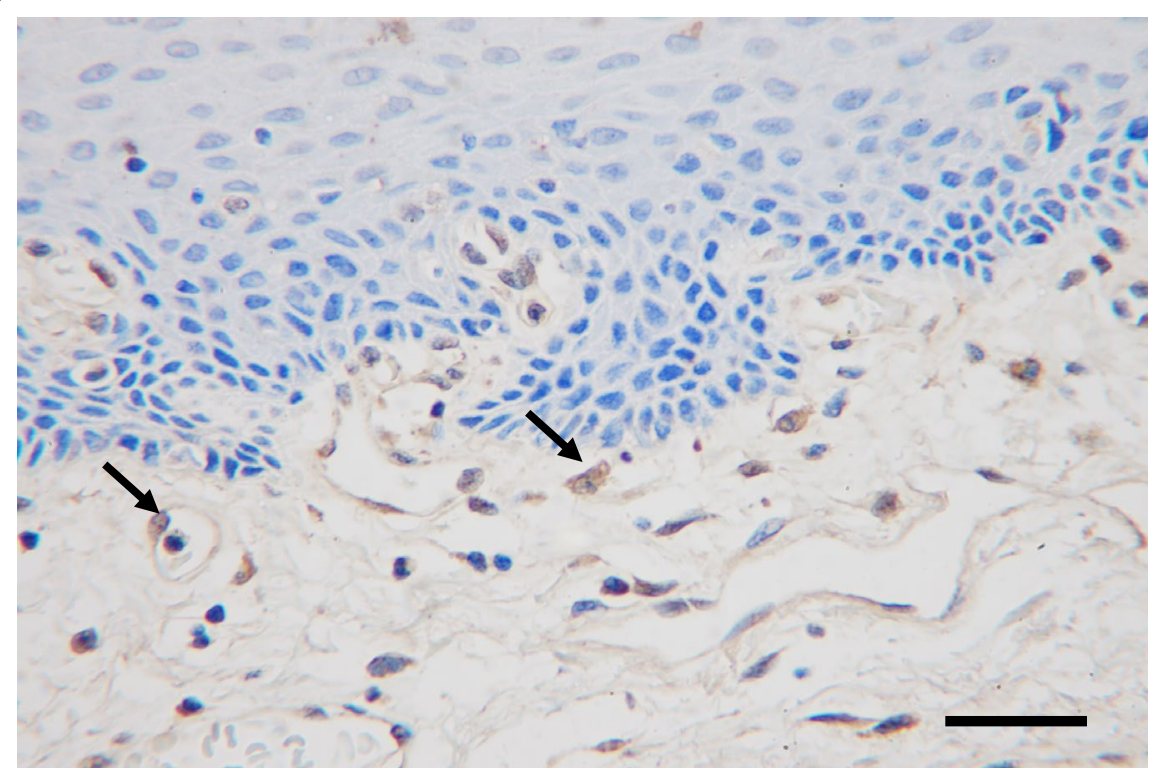

C

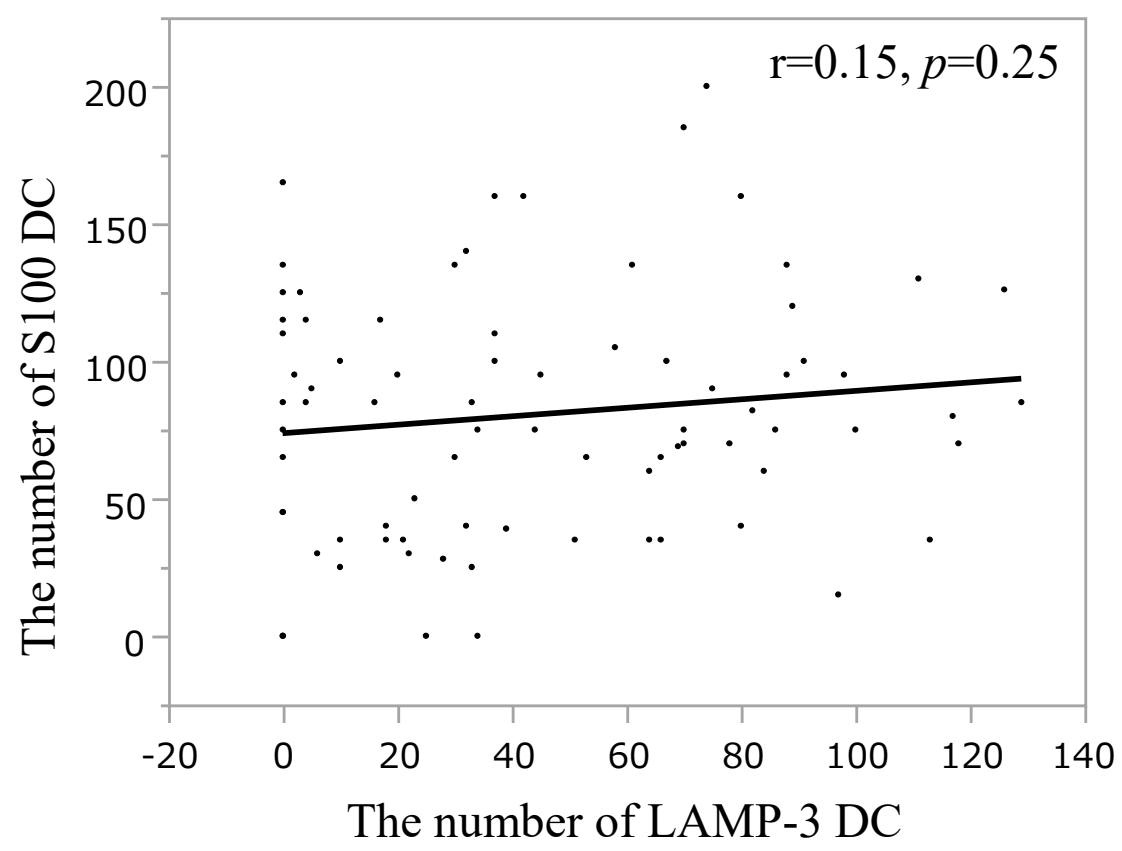




\section{Figure 5}

Dominant stromal types by hematoxylin-eosin staining.

At the most invasive tumor area, stromal type was classified into lymphocyte dominant

type (a) or fibroblast dominant type (b). 
Fig. 5

A

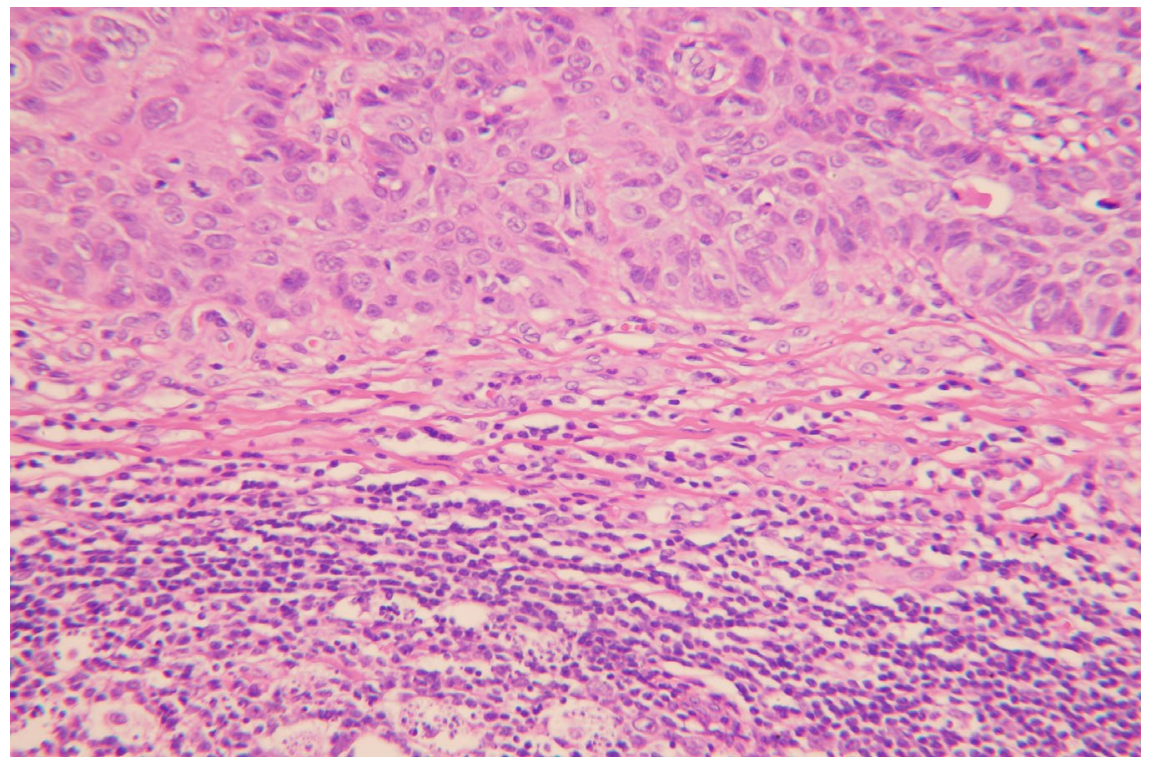

B

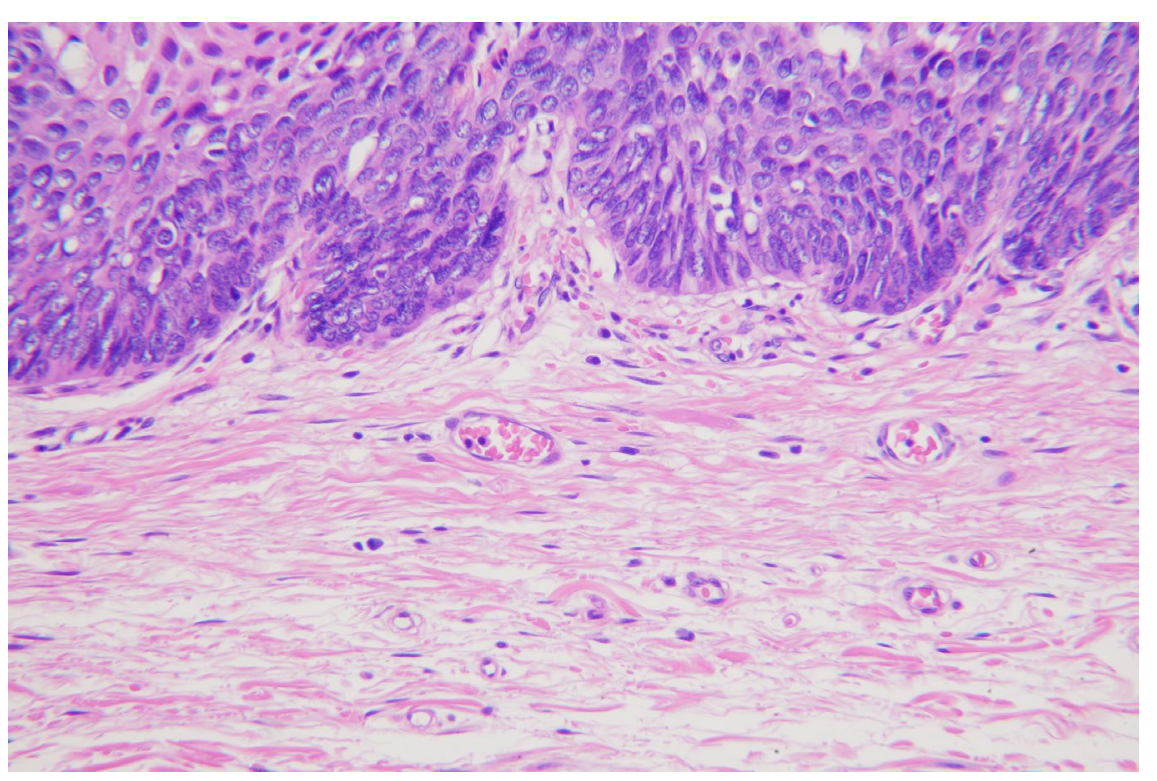




\section{Figure 6}

Kaplan-Meier survival curves according to the LAMP-3 expression in ESCC.

(A) Patients with high LAMP-3 DC staining had a better prognosis than those with low LAMP-3 DC staining in all patients (a: overall survival; $p=0.017$, b: disease free survival; $p=0.043, \log$-rank test). (B) The survival rate of the high intratumoral CD8 T cell group was significantly longer than that of the low intratumoral CD8 T cell group (a: overall survival; $p<0.001$, b: disease free survival; $p<0.001$, log-rank test). (C) The patients were divided into four groups according to the peritumoral LAMP-3 or intratumoral CD8 infiltration intensity (high versus low) in comparison to the other three groups. Among combinations of LAMP-3 and CD8 infiltration, patients with both high LAMP-3 and high CD8 infiltration showed the best prognosis. 


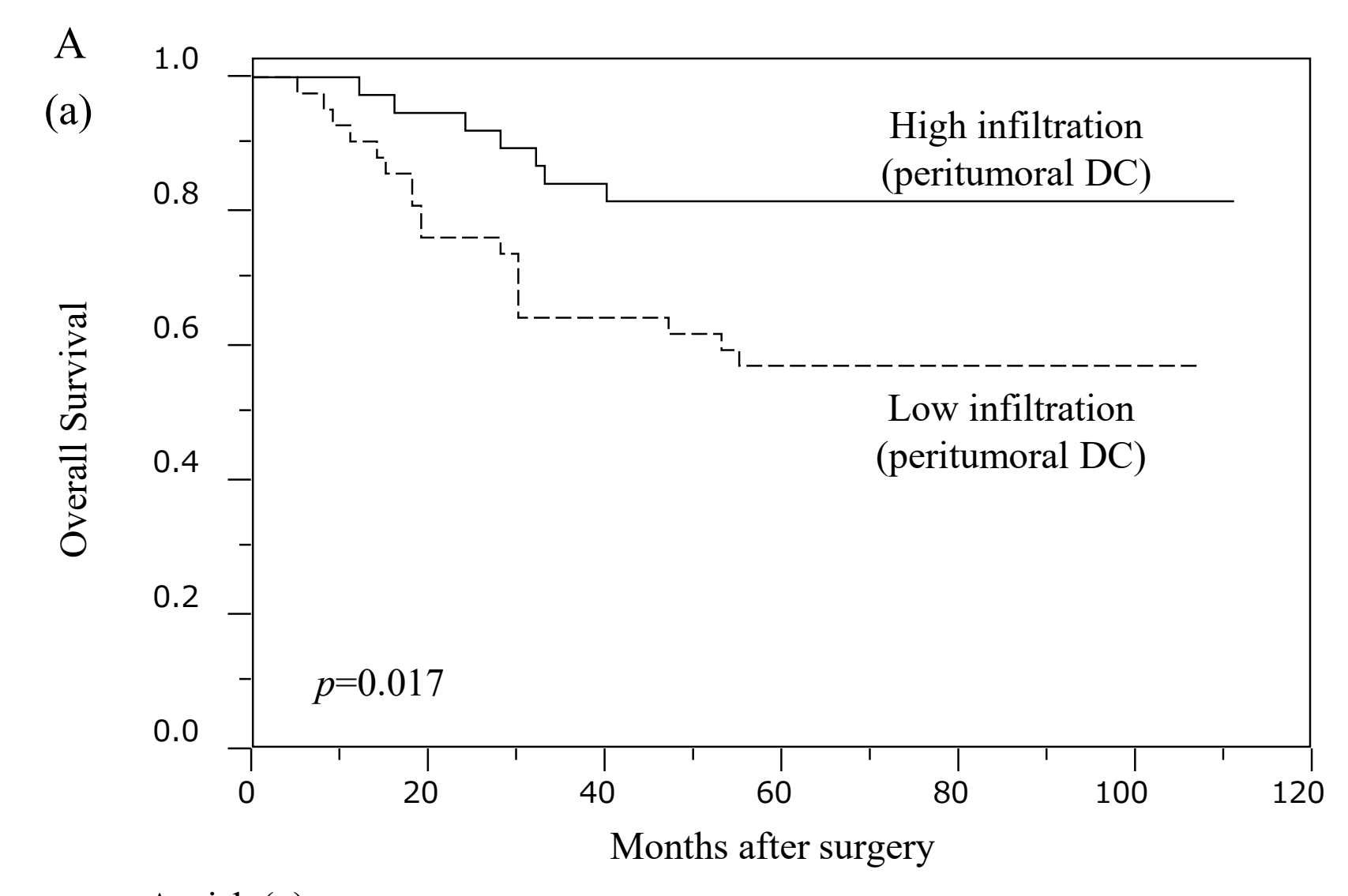

\section{At risk (n)}

$\begin{array}{llllllll}\text { High } & 38 & 37 & 32 & 31 & 8 & 5 & 1 \\ \text { Low } & 42 & 32 & 31 & 23 & 9 & 2 & 0\end{array}$

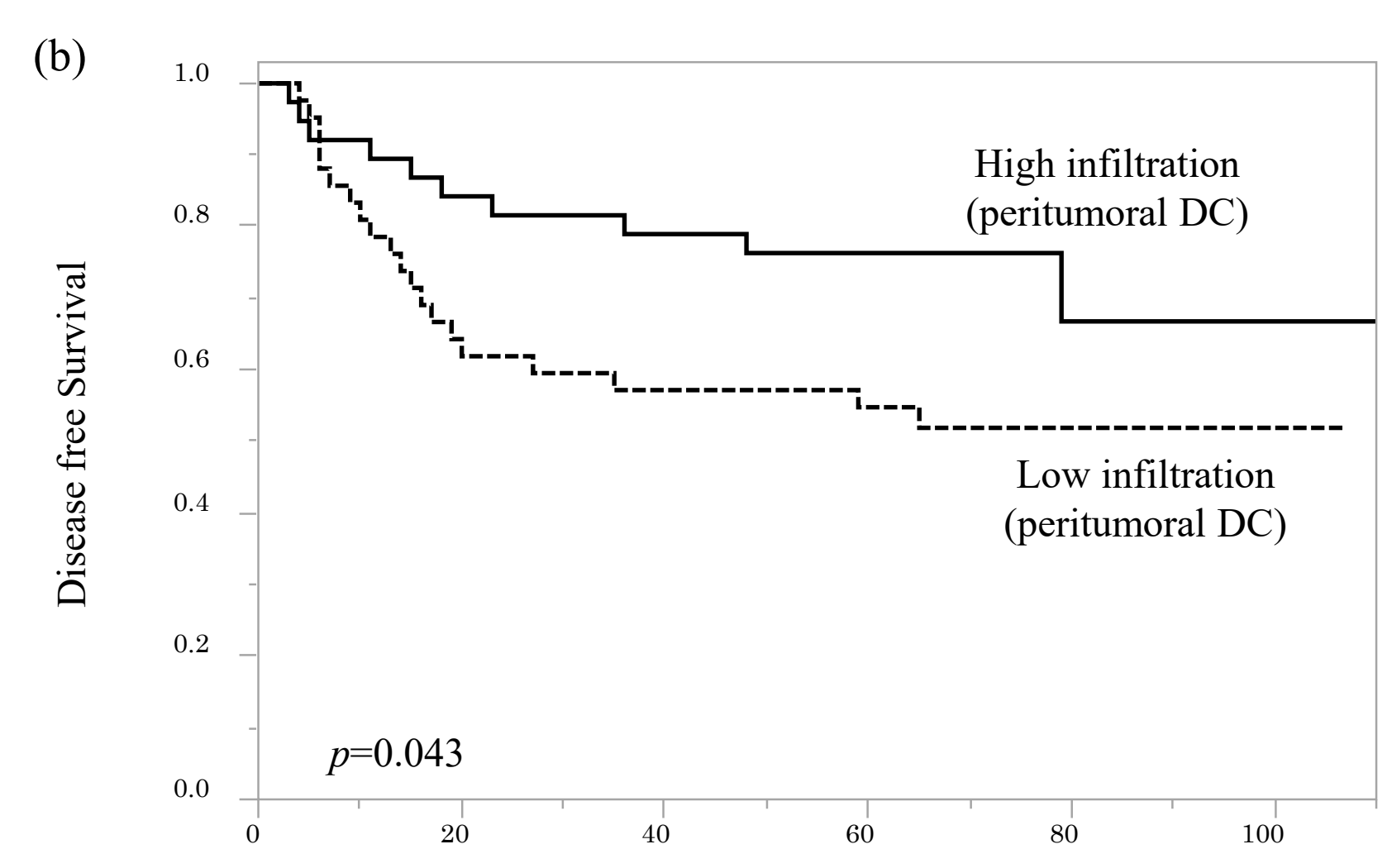

At risk (n)

$\begin{array}{lllllll}\text { High } & 38 & 33 & 31 & 29 & 7 & 4 \\ \text { Low } & 42 & 27 & 25 & 23 & 9 & 2\end{array}$

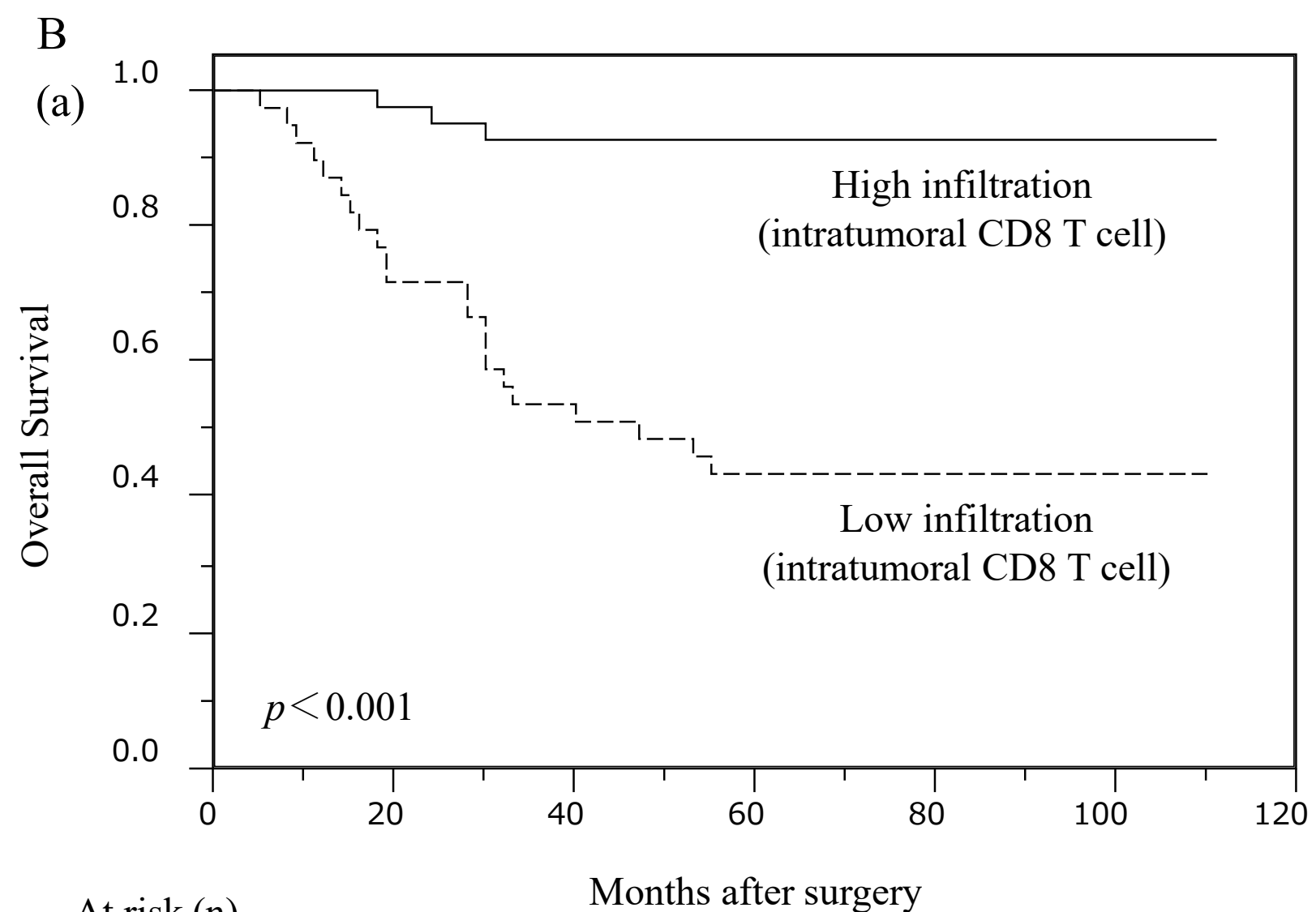

At risk (n)

$\begin{array}{lllllllll}\text { High } & 41 & 41 & 39 & 38 & 13 & 5 & 1\end{array}$

(b)

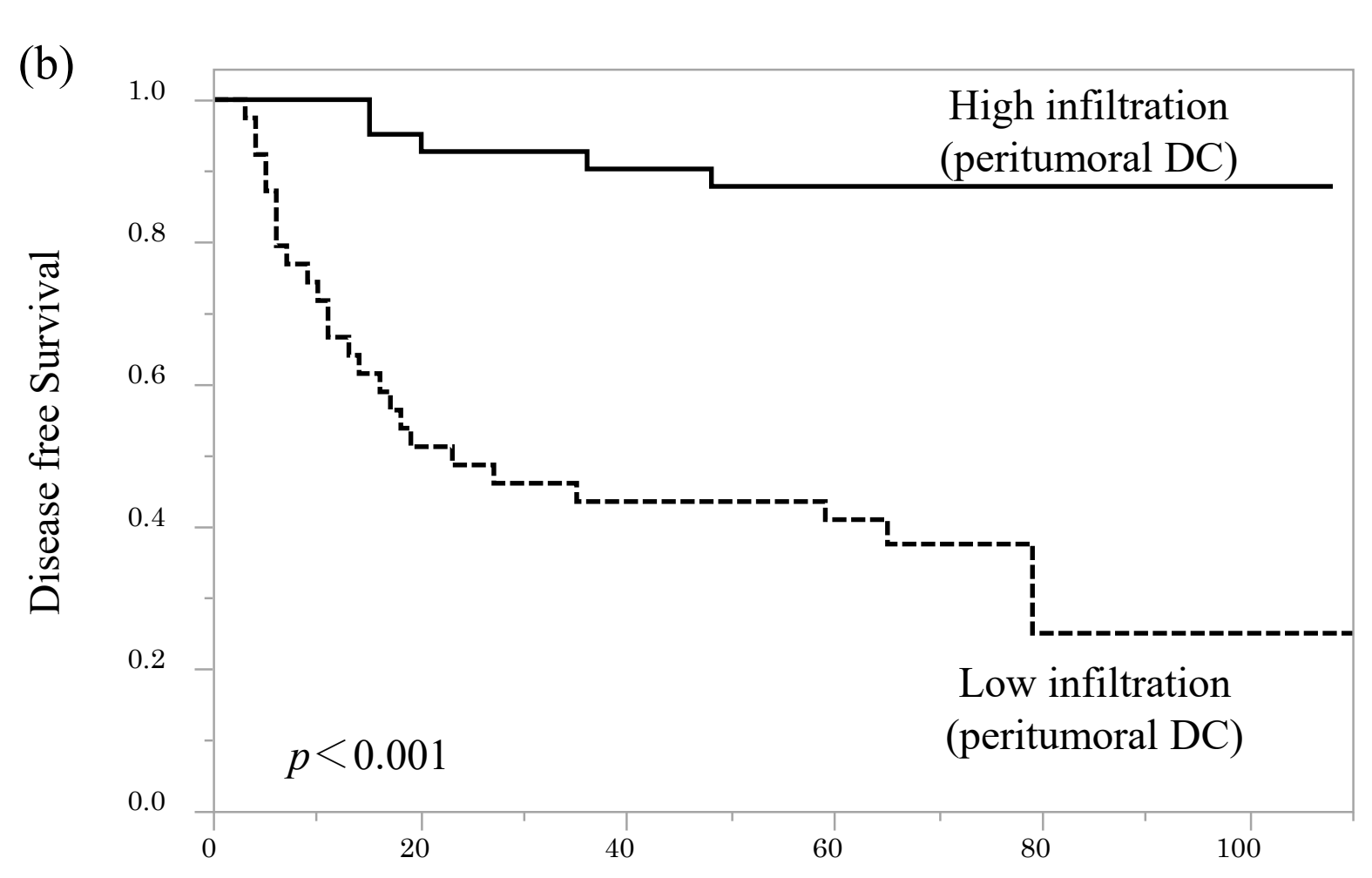

At risk (n)

$\begin{array}{lllllll}\text { High } & 41 & 39 & 38 & 36 & 12 & 54\end{array}$

C

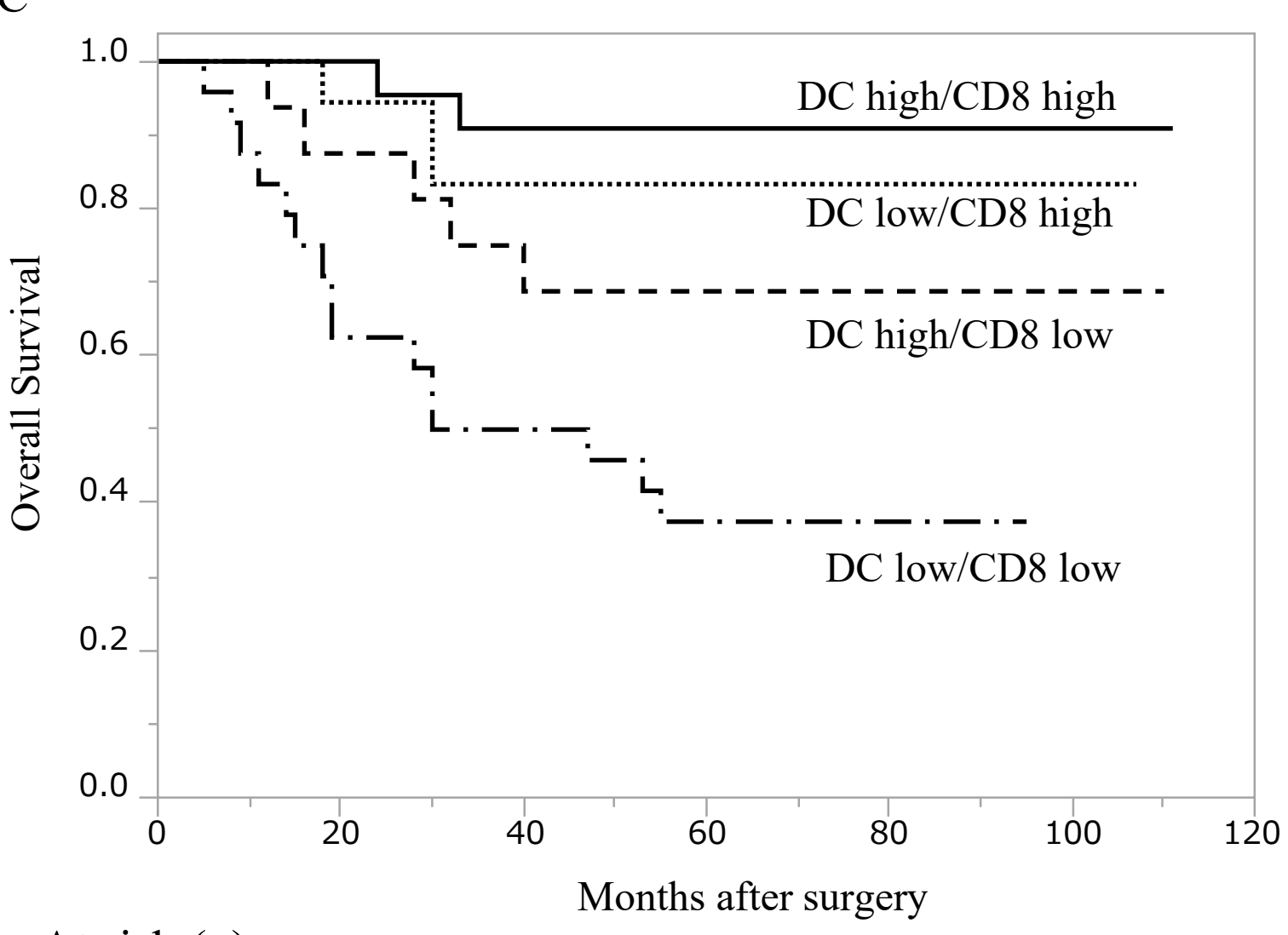

At risk (n)

$\begin{array}{llllllll}\mathrm{DC} \text { high/CD8 high } & 22 & 22 & 20 & 20 & 6 & 3 & 0\end{array}$ $\begin{array}{llllllll}\mathrm{DC} \text { low/CD8 high } & 18 & 17 & 15 & 15 & 5 & 1 & 0\end{array}$ $\begin{array}{llllllll}\mathrm{DC} \text { high/CD8 low } & 16 & 14 & 12 & 11 & 2 & 1 & 0\end{array}$ $\begin{array}{llllllll}\mathrm{DC} \text { low/CD8 low } & 24 & 15 & 12 & 8 & 2 & 0 & 0\end{array}$ 


\section{Figure 7}

Kaplan-Meier survival curves according to the LAMP-3 expression by different tumor depth and pathological stages; A (a): T1, (b) T2-3, B (a) pStage 0-II, (b) pStage III. Patients with high LAMP-3 DC staining had a better prognosis (not significantly) than those with low LAMP-3 DC staining in all these analyses. 
Fig.7

A

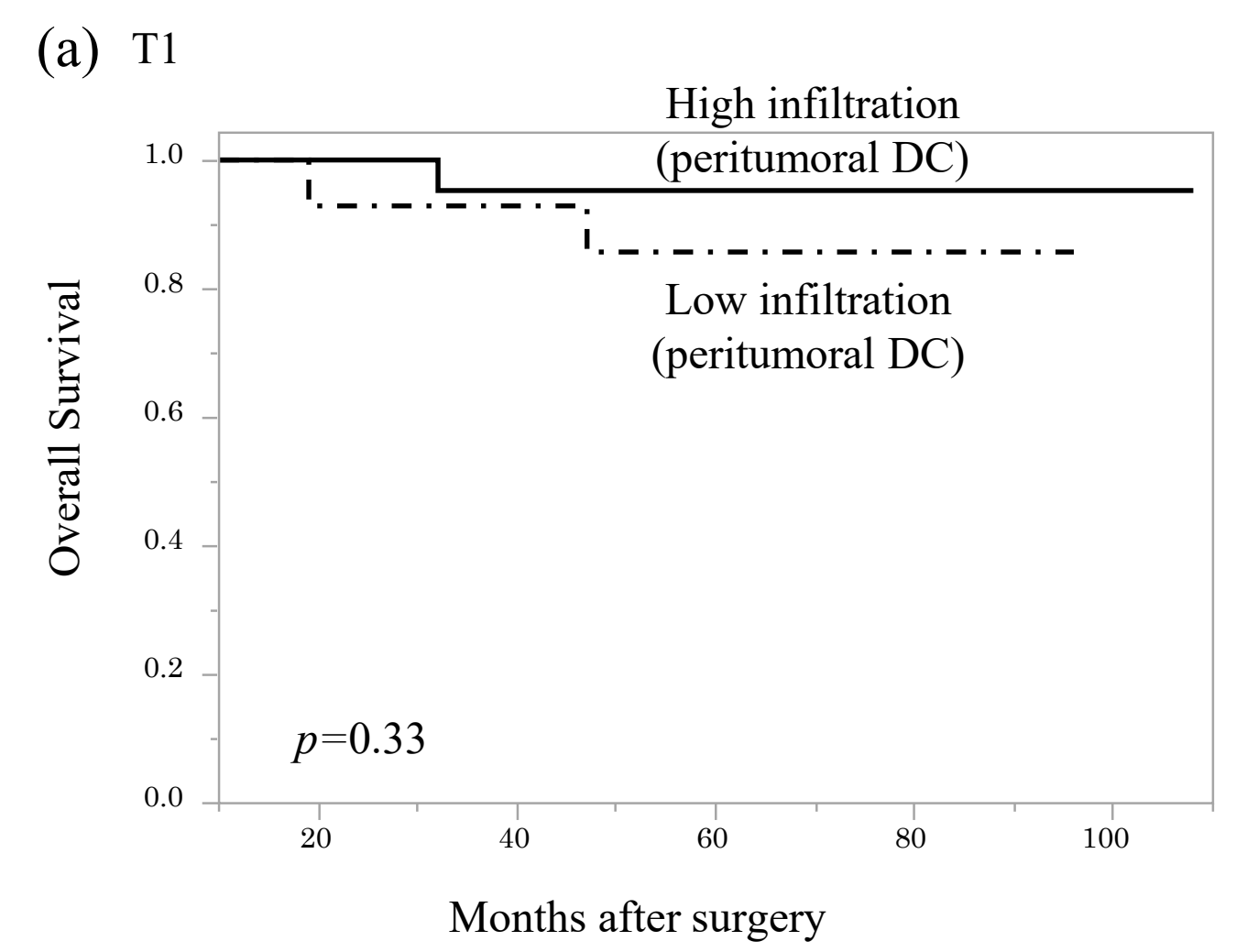

(b) $\mathrm{T} 2-\mathrm{T} 3$

At risk (n)

$\begin{array}{llllclll}\text { High } & 21 & 21 & 21 & 20 & 5 & 3 & 1 \\ \text { Low } & 14 & 13 & 11 & 5 & 1 & 1 & 1\end{array}$

(a) pStage $0-$ II

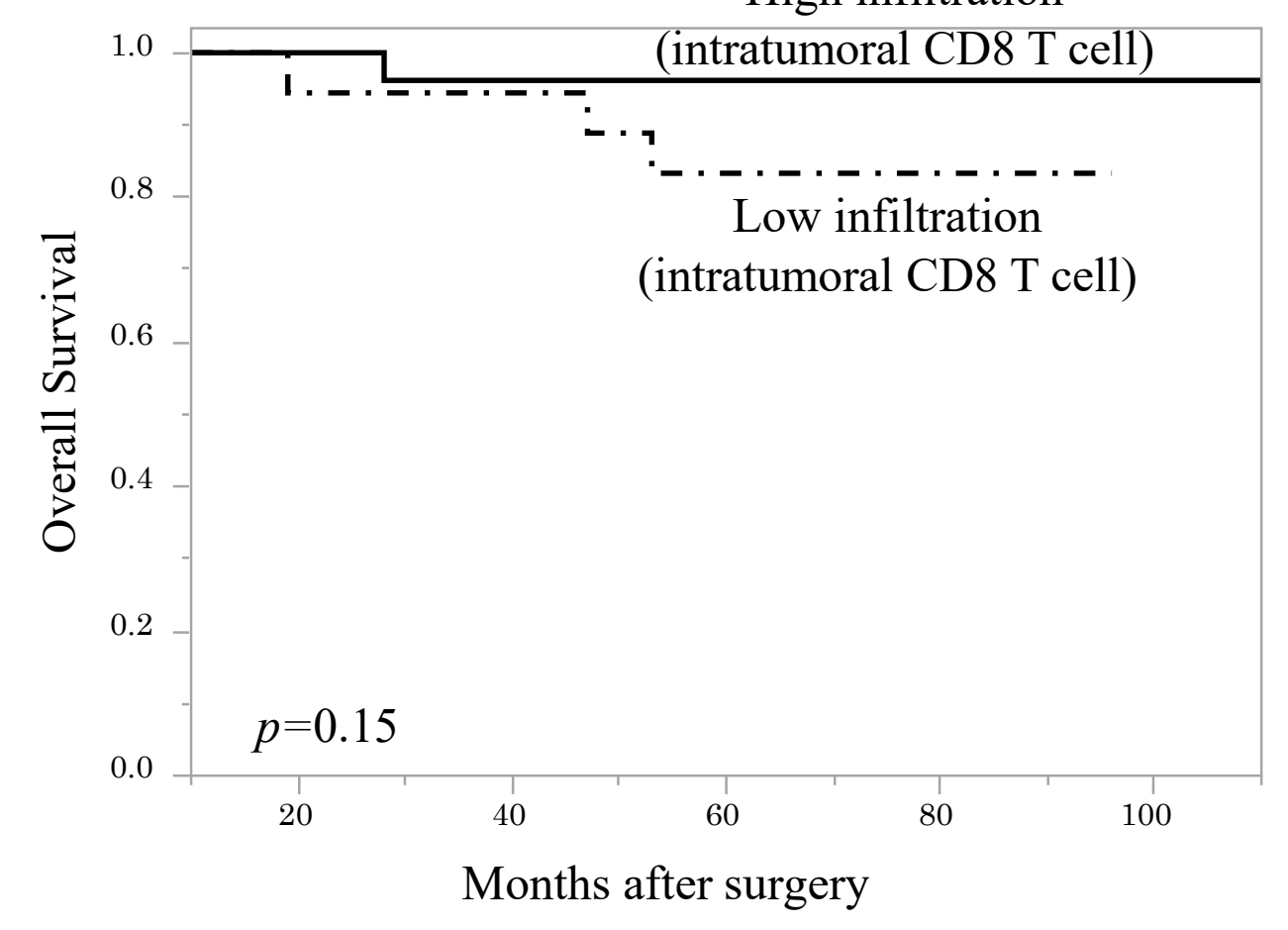

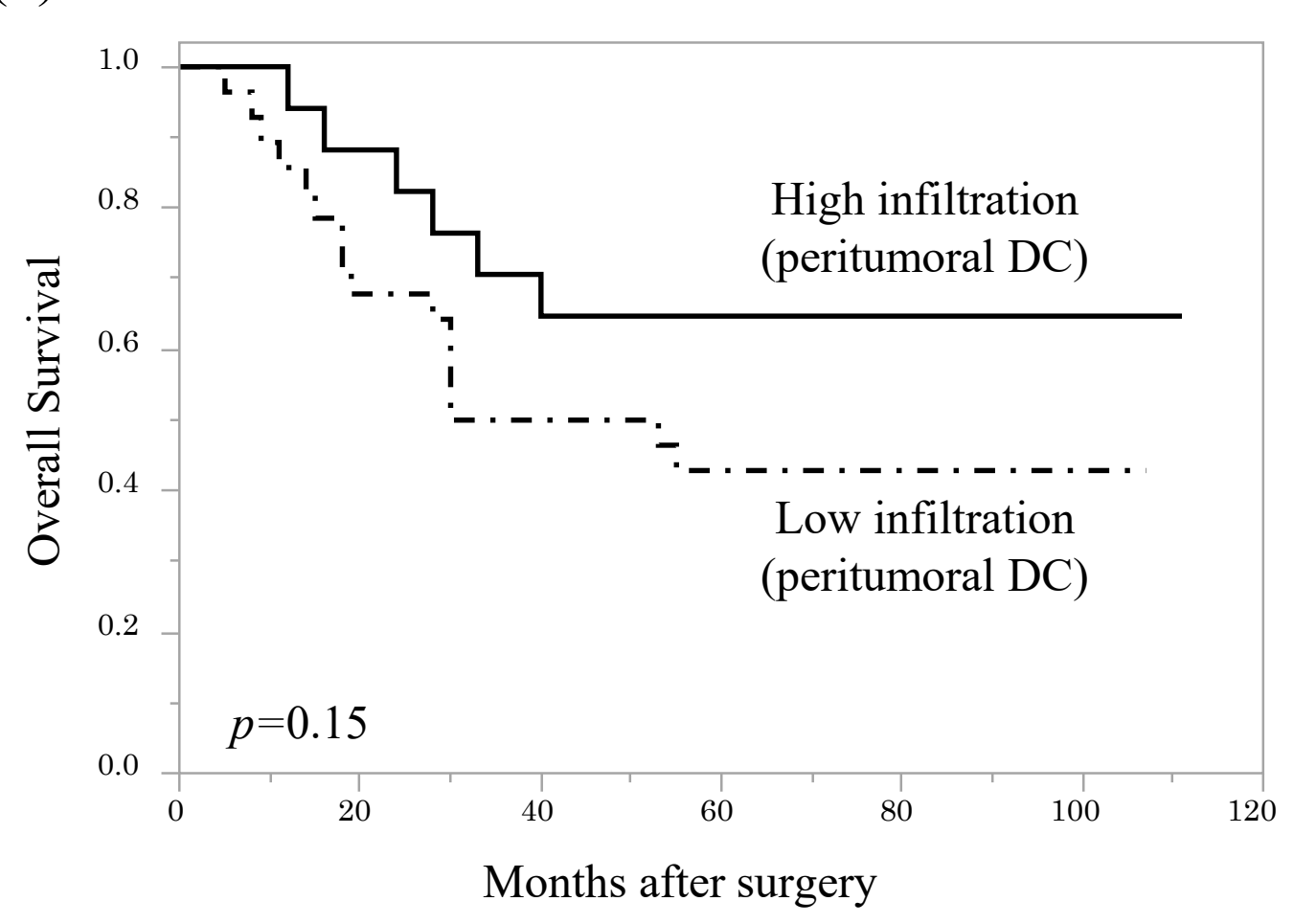

At risk (n)

$\begin{array}{llllllll}\text { High } & 17 & 16 & 12 & 11 & 4 & 3 & 1 \\ \text { Low } & 28 & 20 & 18 & 12 & 4 & 2 & 1\end{array}$

(b) pStageIII

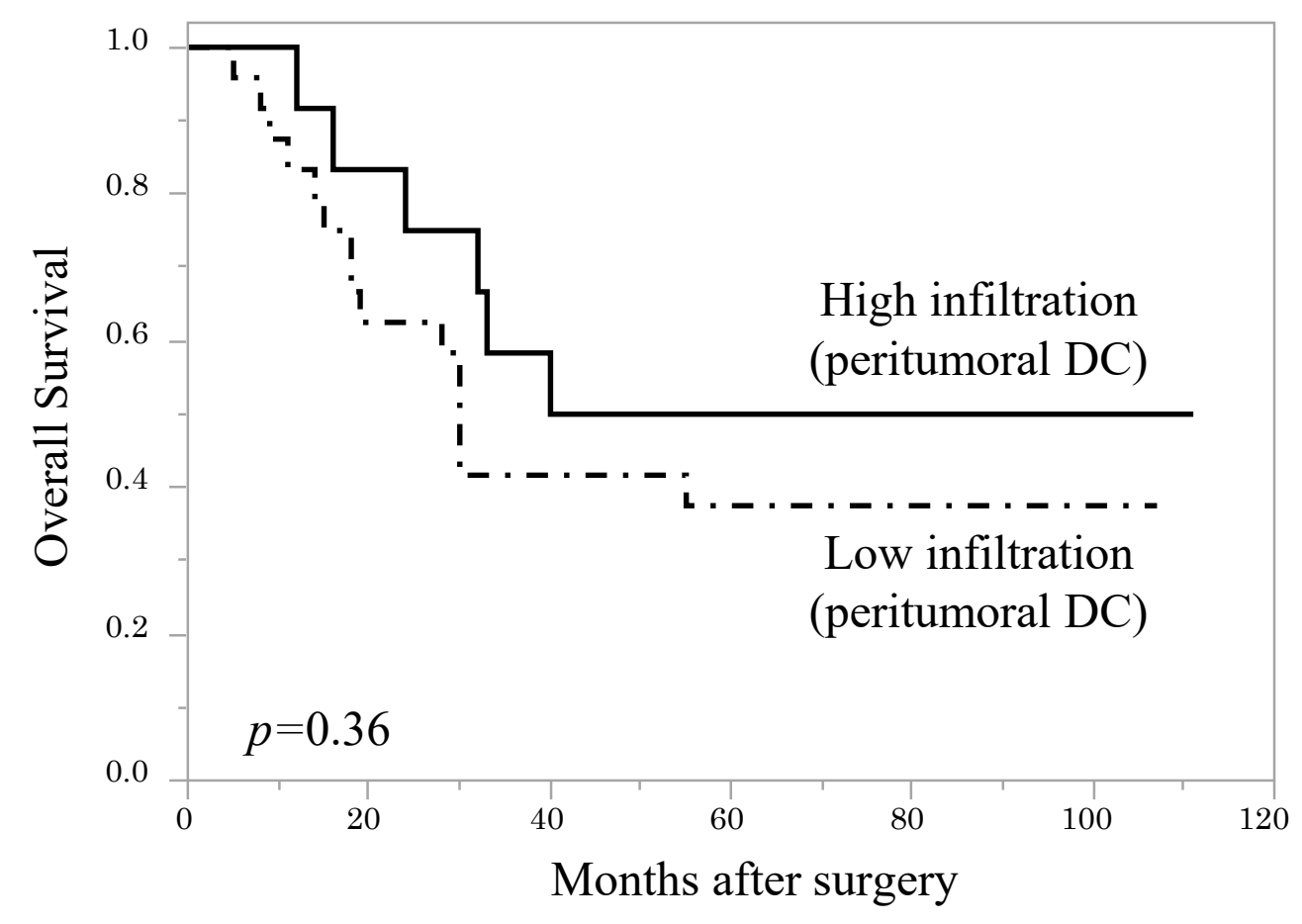

At risk (n)

$\begin{array}{llllllll}\text { High } & 12 & 11 & 7 & 6 & 3 & 2 & 12\end{array}$

$\begin{array}{llllllll}\text { Low } & 24 & 16 & 18 & 12 & 4 & 2 & 1\end{array}$ 